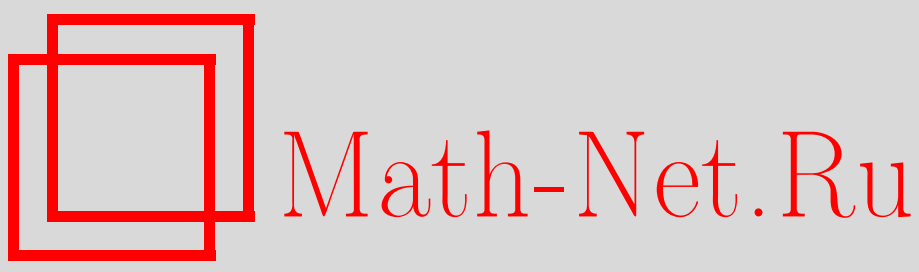

А.-Р. K. Рамазанов, Характеризация полинома наилучшего приближения непрерывной функции со знакочувствительным весом, Матем. сб., 2005, том 196, номер 3, 89-118

DOI: https://doi.org/10.4213/sm1277

Использование Общероссийского математического портала Math-Net.Ru подразумевает, что вы прочитали и согласны с пользовательским соглашением

http://www.mathnet.ru/rus/agreement

Параметры загрузки:

IP : 3.80 .181 .102

26 апреля 2023 г., 12:02:49 
УДК 517.5

\author{
А.-P.К. Рамазанов
}

\title{
Характеризация полинома наилучшего приближения непрерывной функции со знакочувствительным весом
}

\begin{abstract}
Найдены необходимые и достаточные условия полинома наилучшего равномерного приближения непрерьвной функции с произвольным, вообще говоря, неограниченньм знакочувствительным весом; компоненты веса могут принимать также и бесконечные значения, поэтому найденные условия охватьвают, в частности, аптроксимации с интерполяцией в заданных точках и аппроксимации односторонние; в случае веса с единичными компонентами приходим к классической теореме о чебьшёвском альтернансе.

Библиография: 6 названий.
\end{abstract}

\section{Введение}

Знакочувствительным весом на отрезке $\Delta=[a, b]$ называется упорядоченная пара $p=\left(p_{-}, p_{+}\right)$однозначных неотрицательных функций $p_{-}(x)$ и $p_{+}(x)$, определенных на $\Delta$ и принимающих, вообще говоря, также и значение $+\infty$ (см. [1], [2]).

Вес $p$ назовем ограниченным (или непрерывныцм), если таковы обе его компоненты $p_{-}(x)$ и $p_{+}(x)$ на $\Delta$, и неограниченныцм, если на $\Delta$ не ограничена хотя бы одна из этих компонент.

Для определенной на $\Delta$ функции $f(x)$ положим

$$
\begin{gathered}
f^{+}(x)=\max \{f(x), 0\}, \quad f^{-}(x)=(-f(x))^{+}, \\
(f, p)(x)=f^{+}(x) p_{+}(x)-f^{-}(x) p_{-}(x)
\end{gathered}
$$

и $p$-нормой функции $f(x)$ по отрезку $\Delta$ назовем величину

$$
|f|_{p}=|f|_{p, \Delta}=\sup \{|(f, p)(x)|: x \in \Delta\}
$$

$($ считаем $0 \cdot(+\infty)=+\infty \cdot 0=0, c \cdot(+\infty)=+\infty \cdot c=+\infty(-\infty)$ для положительных (отрицательных) чисел $c$ ).

Легко увидеть, что $p$-норма $|f|_{p}=|f|_{p, \Delta}$ в общем случае не является нормой. Она представляет собой положительный сублинейный (т.е. неотрицательный, выпуклый и положительно однородный), вообще говоря, неограниченный функционал на пространстве $M(\Delta)$ ограниченных на $\Delta$ функций:

$$
|f|_{p} \geqslant 0, \quad|f+g|_{p} \leqslant|f|_{p}+|g|_{p}, \quad|c f|_{p}=c|f|_{p}
$$

для любых $f, g \in M(\Delta)$ и неотрицательного числа $c$; однако, вообще говоря, $p$-норма является несимметричной (т.е. возможно неравенство $|-f|_{p} \neq|f|_{p}$ ).

Работа вьполнена при поддержке Российского фонда фундаментальных исследований (грант № 01-01-01019) и Министерства образования РФ. 
Очевидно, для единичного веса (т.е. при $\left.p_{-}(x) \equiv p_{+}(x) \equiv 1\right) p$-норма совпадает с обычной равномерной нормой:

$$
\|f\|_{\Delta}=\sup \{|f(x)|: x \in \Delta\} .
$$

Отметим, что положительные сублинейные функционалы в качестве масштабных функций в конечномерном пространстве рассматривал Г. Минковский [3]. Для веса $p=\left(p_{-}, p_{+}\right)$с непрерывными и строго положительными компонентами $p$-норму в качестве несимметричной нормы рассматривали М. Г. Крейн и А. А. Нудельман [4]; в случае такого веса для полиномиальных приближений функций, непрерывных на отрезке $\Delta$, ими получен аналог теоремы Чебышёва об альтернансе. Е.П. Долженко и Е.А. Севастьянов [1] показали, что наличие (обобщенного) чебышёвского альтернанса является критериальным свойством полинома $Q(x)$ наилучшего приближения непрерывной функции $f(x)$ в $p$-норме для произвольного ограниченного веса $p=\left(p_{-}, p_{+}\right)$, компоненты $p_{-}(x)$ и $p_{+}(x)$ которого являются полунепрерывными сверху (в случае приближения непрерывной функции непрерывными же функциями из некоторого класса достаточно рассматривать только такие веса); при этом в качестве альтернанса они рассматривали точки экстремума функции $(Q-f, p)(x)$, являюшейся прямьм обобшением разности $Q(x)-f(x)$ испытуемого полинома $Q(x)$ и приближаемой функции $f(x)$, рассматриваемой в классической теореме Чебышёва. В [5] получен критерий алгебраического (тригонометрического) полинома наилучшего приближения произвольной ограниченной функции в $p$-норме с произвольным ограниченным весом $p=\left(p_{-}, p_{+}\right)$; в этом случае, вообше говоря, наличие чебьшёвского $p$-альтернанса уже не выступает как единое характеристическое свойство полинома наилучшего приближения.

Ниже рассматривается общий случай приближения непрерывных функций в $p$-норме с произвольным, вообше говоря, разрьвньп и неограниченньм весом $p=$ $\left(p_{-}, p_{+}\right)$на отрезке $\Delta$; равенства $p_{-}(x)=+\infty$ и $p_{+}(x)=+\infty$ также не исключаются. Поэтому нужны новые построения, связанные именно с неограниченностью рассматриваемого веса. Заметим, что введение весов, компоненты которых принимают и значение $\infty$, позволяют рассматривать с единой точки зрения и аппроксимации с интерполяцией в наперед заданных точках, и односторонние аппроксимации.

Основным результатом работы является доказательство необходимого и достаточного условия на алгебраический полином $Q(x)$ для того, чтобы он был полиномом наилучшего приближения для заданной непрерывной функции $f(x)$ относительно заданного знакочувствительного веса $p(x)$, вообше говоря, разрывного, неограниченного и принимающего значение $+\infty$ (теорема 1 - если степень $Q(x)$ меньше мощности множества точек одновременной неограниченности компонент $p_{-}(x)$ и $p_{+}(x)$ веса $p(x)$, и теорема 4 - если степень $Q(x)$ не менњше мощности этого множества).

\section{§1. Обозначения и уточнение постановки задачи}

Пусть функция $f(x)$ непрерьвна на отрезке $\Delta=[a, b]$, на котором определен вес $p=\left(p_{-}, p_{+}\right) ; \mathscr{P}_{n}-$ множество всех алгебраических полиномов степени не выше $n$, $n=0,1, \ldots$, с действительными коэффициентами.

Наименьшее полиномиальное уклонение (наилучшее приближение) порядка $n$ 
функции $f(x)$ в $p$-норме определим равенством

$$
E_{n}(f, p, \Delta)=\inf \left\{|P-f|_{p, \Delta}: P \in \mathscr{P}_{n}\right\}, \quad n=0,1, \ldots
$$

Если для некоторого полинома $Q \in \mathscr{P}_{n}$ выполняется равенство

$$
|Q-f|_{p, \Delta}=E_{n}(f, p, \Delta),
$$

то $Q$ назовем полиномом наилучшего приближения порядка $n$ функции $f(x)$ в $p$-норме или с весом $p=\left(p_{-}, p_{+}\right)$на отрезке $\Delta$.

Через $\Delta_{-}^{\infty}\left(\Delta_{+}^{\infty}\right)$ обозначим множество всех тех точек $x$ отрезка $\Delta$, в любой окрестности каждой из которых не ограничена компонента $p_{-}(x)$ (соответственно $\left.p_{+}(x)\right)$ или для которых $p_{-}(x)=+\infty\left(p_{+}(x)=+\infty\right)$.

Предварительно рассмотрим случай, когда для данного веса $p=\left(p_{-}, p_{+}\right)$мощность множества $F=\Delta_{-}^{\infty} \cap \Delta_{+}^{\infty}$ превосходит порядок наилучшего полиномиального приближения данной функции в $p$-норме.

ТЕОРема 1. Пусть на отрезке $\Delta=[a, b]$ задань произвольныи вес $p=$ $\left(p_{-}, p_{+}\right)$и непрерывная функиия $f(x)$; для данного $n, \quad n=0,1, \ldots$, полином $Q \in \mathscr{P}_{n}, L(x, f, T)$ обозначает интерполяционный полином степени не выше $n$ функиии $f(x)$ с множеством узлов $T$; мощность множества $F=\Delta_{-}^{\infty} \cap \Delta_{-}^{\infty}$ больие $n$.

Тогда для выполнения равенства (1) с данным полиномом $Q(x)$ необходимо и достаточно существование такого набора различных точек $T=$ $\left\{x_{0}, x_{1}, \ldots, x_{n}\right\} \subset F$, что $L(x, f, T) \equiv Q(x)$ или $|L(\cdot, f, T)-f|_{p, \Delta}=\infty$.

ДокАЗАТЕльство. Пусть для полинома $Q \in \mathscr{P}_{n}$ выполнено равенство (1). Будем считать $|\cdot|_{p}=|\cdot|_{p, \Delta}$ и рассмотрим два случая.

1) $|Q-f|_{p}<\infty$. Тогда при любом $x \in F$ имеем $Q(x)=f(x)$; в частности, $Q(x)=f(x), x \in T$ для любого набора различных точек $T=\left\{x_{0}, x_{1}, \ldots, x_{n}\right\} \subset F$, а поэтому $L(x, f, T) \equiv Q(x)$ для каждого такого набора $T$.

2) $|Q-f|_{p}=\infty$. Тогда $E_{n}(f, p, \Delta)=\infty$, поэтому для любого полинома степени не выше $n$, в частности для $L(x, f, T)$, при любом выборе $T=\left\{x_{0}, x_{1}, \ldots, x_{n}\right\}$ различных точек из $F$ получим $|L(\cdot, f, T)-f|_{p}=\infty$.

Необходимость доказана, докажем достаточность.

Пусть сушествует набор различных точек $T=\left\{x_{0}, x_{1}, \ldots, x_{n}\right\} \subset F \mathrm{c} L(x, f, T) \equiv$ $Q(x)$. Тогда $Q(x)=f(x)$ при $x \in T$.

Если при этом $|Q-f|_{p}<\infty$, то для любого другого полинома $P \in \mathscr{P}_{n}, P \neq Q$, имеем $|P-f|_{p}=\infty$.

Действительно, в противном случае $P(x)=f(x)$ при $x \in F$, откуда следует $P(x)=f(x)=Q(x)$ при $x \in T$, а поэтому $P(x) \equiv Q(x)$.

Следовательно, для полинома $Q(x)$ выполняется равенство (1).

Пусть теперь $|Q-f|_{p}=\infty$. В этом случае исследуемое первое $Q(x) \equiv L(x, p, T)$ из условий теоремы приводит ко второму, а поэтому остается рассмотреть случай $|L(\cdot, f, T)-f|_{p}=\infty$. Тогда $E_{n}(f, p, \Delta)=\infty$, так как величина $|P-f|_{p}$ при $P \in \mathscr{P}_{n}$ и мощности множества $F$ большей $n$ может быть конечной лишш для $P(x) \equiv L(x, f, T)$.

Следовательно, для любого полинома $P \in \mathscr{P}_{n}$ (вместо $Q(x)$ ), в частности самого полинома $Q(x)$, выполняется равенство (1) и теорема 1 доказана. 
ЗАмЕчАНИЕ 1. Как следует из теоремы 1 , интерполяционный полином $L(x, f, T)$ является полиномом наилучшего приближения порядка $n$ функции $f(x)$ в $p$-норме, однако, как следует из приведенного доказательства, интерполяционность не является необходимым условием полинома наилучшего приближения.

ЗАмечание 2. Если для данного веса $p=\left(p_{-}, p_{+}\right)$, как и выше, мошность множества $F=\Delta_{-}^{\infty} \cap \Delta_{+}^{\infty}$ больше $n, n=0,1, \ldots$, то для любых двух различных полиномов $P, Q \in \mathscr{P}_{n}$, очевидно, имеем $|P-Q|_{p}=\infty$. Отсюда вытекает еше одно достаточное условие полинома наилучшего приближения в рассматриваемом случае: если для данного полинома $Q \in \mathscr{P}_{n}$ и функции $f(x)$ величина $|-(Q-f)|_{p}=|f-Q|_{p}$ конечна, то выполняется равенство (1) (так как тогда из неравенства $|P-Q|_{p} \leqslant|P-f|_{p}+|f-Q|_{p}$ имеем $|P-f|_{p}=\infty$ при любом другом полиноме $\left.P \in \mathscr{P}_{n}\right)$.

Отметим также, что если функция $f(x)$ непрерывна на $\Delta$, полином $Q \in \mathscr{P}_{n}$, $n=0,1, \ldots$, и существует точка $x \in \Delta_{-}^{\infty}$ с $Q(x)<f(x)$ или существует точка $x \in \Delta_{+}^{\infty}$ с $Q(x)>f(x)$, то $|Q-f|_{p}=\infty$, а следовательно, для этого случая равенство (1) выполняется тогда и только тогда, когда $E_{n}(f, p, \Delta)=\infty$.

Поэтому всюду ниже, если не оговорено противное, будем считать, что функция $f(x)$ непрерьвна на отрезке $\Delta=[a, b]$, полином $Q \in \mathscr{P}_{n}, n=0,1, \ldots, Q(x) \geqslant f(x)$ при $x \in \Delta_{-}^{\infty}, Q(x) \leqslant f(x)$ при $x \in \Delta_{+}^{\infty}$ и, в частности, $Q(x)=f(x)$ при $x \in F$, мошность множества $F$ не больше $n$ для данного на $\Delta$ веса $p=\left(p_{-}, p_{+}\right)$.

Через $\Delta\left(p_{-}\right)$и $\Delta\left(p_{+}\right)$обозначим множества соответственно $(-)$- и $(+)$-точек $p$-экстремума разности $Q(x)-f(x)$ на отрезке $\Delta$, т.е. таких точек $y \in \Delta$, что при $E=(y-\delta, y+\delta) \cap \Delta$ и любом $\delta>0$ выполняются соответственно равенства

$$
\begin{aligned}
& \sup \left\{[Q(x)-f(x)]^{-} p_{-}(x): x \in E\right\}=|Q-f|_{p, \Delta}, \\
& \sup \left\{[Q(x)-f(x)]^{+} p_{+}(x): x \in E\right\}=|Q-f|_{p, \Delta} .
\end{aligned}
$$

Если равенства (2) и (3) вьполняются при $E=(y-\delta, y] \cap \Delta(E=[y, y+\delta) \cap \Delta)$, то будем говорить, что точка $y$ принадлежит $\Delta\left(p_{-}\right)$и соответственно $\Delta\left(p_{+}\right)$относительно своих левых (правых) окрестностей.

Через $\Delta\left(p_{-}, n\right)$ обозначим множество точек $y \in \Delta \backslash \Delta\left(p_{-}\right)$, для которых при сколь угодно малых $\delta>0$ и $\alpha>0$ найдется точка $x \in(y-\delta, y+\delta) \cap \Delta$ c

$$
\left(f(x)-Q(x)+\alpha|x-y|^{n}\right) p_{-}(x) \geqslant|Q-f|_{p, \Delta} .
$$

Если при аналогичных $\delta, \alpha, x$ для $y \in \Delta \backslash \Delta\left(p_{+}\right)$вместо (4) выполняется неравенство

$$
\left(Q(x)-f(x)+\alpha|x-y|^{n}\right) p_{+}(x) \geqslant|Q-f|_{p, \Delta},
$$

то по определению точка $y \in \Delta\left(p_{+}, n\right)$. При этом точка $y \in \Delta\left(p_{-}, n\right)$ или точка $y \in$ $\Delta\left(p_{+}, n\right)$ относительно своих левых (правых) окрестностей, если неравенство (4) или соответственно (5) выполняется при $x \in(y-\delta, y] \cap \Delta(x \in[y, y+\delta) \cap \Delta)$.

Определим также множества

$$
\begin{aligned}
& \Delta_{-}^{\infty}(Q=f)=\left\{x \in \Delta_{-}^{\infty} \backslash \Delta_{+}^{\infty}: Q(x)=f(x)\right\} \\
& \Delta_{+}^{\infty}(Q=f)=\left\{x \in \Delta_{+}^{\infty} \backslash \Delta_{-}^{\infty}: Q(x)=f(x)\right\}
\end{aligned}
$$


и множества $\Delta_{-}^{\infty}(Q=f)^{\prime}, \Delta_{+}^{\infty}(Q=f)^{\prime}$ их предельных точек; при этом, как и вьше, скажем, что точка $y \in \Delta_{-}^{\infty}(Q=f)^{\prime}$ или точка $y \in \Delta_{+}^{\infty}(Q=f)^{\prime}$ относительно своих левых (правых) окрестностей, если она служит предельной точкой пересечения промежутка $(y-\delta, y]([y, y+\delta))$ соответственно с $\Delta_{-}^{\infty}(Q=f)$ или c $\Delta_{+}^{\infty}(Q=f)$.

Для сокрашения записи всюду ниже положим

$$
\begin{aligned}
& E_{-}=\Delta\left(p_{-}\right) \cup \Delta\left(p_{-}, n\right) \cup \Delta_{-}^{\infty}(Q=f)^{\prime}, \\
& G_{-}=\Delta\left(p_{-}\right) \cup \Delta\left(p_{-}, n\right) \cup \Delta_{-}^{\infty}(Q=f) ;
\end{aligned}
$$

заменив в правых частях для $E_{-}$и $G_{-}$все знаки - на знаки + , получим соответственно множества $E_{+}$и $G_{+}$; будем считать также $|\cdot|_{p}=|\cdot|_{p, \Delta}$.

\section{$\S 2$. Первое достаточное условие полинома наилучшего приближения}

Будем придерживаться обозначений, принятых вьше; в частности, на отрезке $\Delta$ для данных веса $p=\left(p_{-}, p_{+}\right)$, функции $f(x)$ и полинома $Q(x)$ множества $E_{-}$и $E_{+}$ определяются так же, как и в $\S 1$.

Теорема 2. Пусть на отрезке $\Delta$ заданы вес $p=\left(p_{-}, p_{+}\right)$и непрерьвная функция $f(x)$, алгебраический полином $Q(x)$ степени $\leqslant n, n=0,1, \ldots$, удовлетворяет неравенству $Q(x) \geqslant f(x)$ при $x \in \Delta_{-}^{\infty}$ и неравенству $Q(x) \leqslant f(x)$ nри $x \in \Delta_{+}^{\infty}$.

Если множество $E_{-} \cap E_{+}$содержит хотя бь одну точку относительно ее односторонних окрестностей, то $Q(x)$ является полиномом наилучшего приближения порядка $n$ функции $f(x)$ в р-норме с весом $p=\left(p_{-}, p_{+}\right)$на отрезке $\Delta$.

ДокАЗАТЕЛЬСтво. Так как при $|Q-f|_{p}=0$ равенство (1), очевидно, выполняется, будем считать $|Q-f|_{p}>0$.

Пусть некоторая точка $x_{0} \in E_{-} \cap E_{+}$относительно ее, например, правых окрестностей.

Тогда эта точка $x_{0} \in F=\Delta_{-}^{\infty} \cap \Delta_{+}^{\infty}$.

Действительно, допустим, что $x_{0} \notin \Delta_{+}^{\infty}$. Тогда из $x_{0} \in E_{+}$следует $x_{0} \in \Delta\left(p_{+}\right)$, а поэтому $Q\left(x_{0}\right)>f\left(x_{0}\right)$. В силу непрерьвности $Q(x)$ и $f(x)$ найдется такое $\delta>0$, что при $x \in\left[x_{0}-\delta, x_{0}+\delta\right]$ имеем $Q(x)>f(x)$.

Отсюда, очевидно, следует, что $x_{0} \notin \Delta\left(p_{-}\right)$, так как $|Q-f|_{p}>0$.

Далее, $x_{0} \notin \Delta_{-}^{\infty}(Q=f)^{\prime}$, так как в противном случае по непрерывности получили бы $Q\left(x_{0}\right)=f\left(x_{0}\right)$.

Наконец, так как

$$
\max \left\{f(x)-Q(x): x \in\left[x_{0}-\delta, x_{0}+\delta\right]\right\}<0,
$$

условие (4) также не может вьполняться и $x_{0} \notin \Delta\left(p_{-}, n\right)$.

Следовательно, допушение $x_{0} \notin \Delta_{+}^{\infty}$ приводит к противоречию с $x_{0} \in E_{-}$, а поэтому $x_{0} \in \Delta_{+}^{\infty}$.

Аналогично показываем, что $x_{0} \in \Delta_{-}^{\infty}$. 
Так как $x_{0} \in F$, то выполняется равенство $Q\left(x_{0}\right)=f\left(x_{0}\right)$.

Допустим теперь, что сушествует полином $P \in \mathscr{P}_{n}, n=0,1, \ldots$, такой, что

$$
|P-f|_{p}<|Q-f|_{p}
$$

Тогда $|P-f|_{p}$ конечна, а поэтому из $x_{0} \in F$ имеем $P\left(x_{0}\right)=f\left(x_{0}\right)$.

Следовательно, выполняется равенство $Q\left(x_{0}\right)=P\left(x_{0}\right)$.

Пусть сначала точка $x_{0} \in \Delta\left(p_{-}\right) \cap \Delta\left(p_{+}\right)$(относительно ее правых окрестностей). Тогда найдутся две последовательности точек $x_{j} \rightarrow x_{0}, j \rightarrow \infty, x_{j} \geqslant x_{0}$, и $y_{j} \rightarrow x_{0}, j \rightarrow \infty, y_{j} \geqslant x_{0}$, такие, что при $j \rightarrow \infty$ обе последовательности $\left(f\left(x_{j}\right)-Q\left(x_{j}\right)\right) p_{-}\left(x_{j}\right)$ и $\left(Q\left(x_{j}\right)-f\left(x_{j}\right)\right) p_{+}\left(x_{j}\right)$ имеют пределом $|Q-f|_{p}>0$.

Если теперь $Q(x)>P(x)$ при $x \in\left(x_{0}, x_{0}+\delta\right)$ с некоторым $\delta>0$, то при $j \rightarrow \infty$ получим

$$
|P-f|_{p} \geqslant\left(f\left(x_{j}\right)-P\left(x_{j}\right)\right) p_{-}\left(x_{j}\right) \geqslant\left(f\left(x_{j}\right)-Q\left(x_{j}\right)\right) p_{-}\left(x_{j}\right) \rightarrow|Q-f|_{p} .
$$

Если же $Q(x)<P(x)$ при $x \in\left(x_{0}, x_{0}+\delta\right)$ с некоторьм $\delta>0$, то при $j \rightarrow \infty$ также получим

$$
|P-f|_{p} \geqslant\left(P\left(y_{j}\right)-f\left(y_{j}\right)\right) p_{+}\left(y_{j}\right) \geqslant\left(Q\left(y_{j}\right)-f\left(y_{j}\right)\right) p_{+}\left(y_{j}\right) \rightarrow|Q-f|_{p} .
$$

Оба раза пришли к противоречию с допушением (6).

Следовательно, если некоторая точка $x_{0} \in \Delta\left(p_{-}\right) \cap \Delta\left(p_{+}\right)$относительно ее правых окрестностей, то выполняется равенство (1).

Рассмотрим теперь случай точки $x_{0} \in \Delta\left(p_{-}\right) \cap \Delta\left(p_{+}, n\right)$ относительно ееправых окрестностей.

Так как $x_{0} \in \Delta\left(p_{-}\right)$и вьполняется (6), сушествует последовательность $x_{j} \rightarrow x_{0}$, $j \rightarrow \infty, x_{j} \geqslant x_{0}$, такая, что

$$
\left(Q\left(x_{j}\right)-f\left(x_{j}\right)\right) p_{-}\left(x_{j}\right)>|P-f|_{p}
$$

для всех достаточно больших номеров $j$ (для них не исключается случай $x_{j}=x_{0}$ ). Тогда

$$
\begin{aligned}
& \left(Q\left(x_{j}\right)-f\left(x_{j}\right)\right)^{-} p_{-}\left(x_{j}\right) \leqslant\left(Q\left(x_{j}\right)-P\left(x_{j}\right)\right)^{-} p_{-}\left(x_{j}\right)+|P-f|_{p} ; \\
& \left(Q\left(x_{j}\right)-P\left(x_{j}\right)\right)^{-} p_{-}\left(x_{j}\right) \geqslant\left(Q\left(x_{j}\right)-f\left(x_{j}\right)\right)^{-} p_{-}\left(x_{j}\right)-|P-f|_{p}>0 .
\end{aligned}
$$

Поэтому $Q\left(x_{j}\right)-P\left(x_{j}\right)<0$ при всех достаточно больших $j$, отсюда $Q(x)<P(x)$ при $x \in\left(x_{0}, x_{0}+\delta\right)$ с некоторым достаточно мальм $\delta>0$.

В нашем случае $Q\left(x_{0}\right)=P\left(x_{0}\right)$, поэтому сушествует $a_{m} \neq 0,1 \leqslant m \leqslant n$, и

$$
P(x)-Q(x)=a_{m}\left(x-x_{0}\right)^{m}+a_{m+1}\left(x-x_{0}\right)^{m+1}+\cdots+a_{n}\left(x-x_{0}\right)^{n}>0
$$

при $x \in\left(x_{0}, x_{0}+\delta\right)$.

Возьмем $0<\alpha<(1 / 2)\left|a_{m}\right|$. Тогда при достаточно малых $\delta>0$ для $x \in$ $\left(x_{0}, x_{0}+\delta\right)$ получим

$$
P(x)-Q(x) \geqslant \alpha\left|x-x_{0}\right|^{n} .
$$


Теперь из того, что $x_{0} \in \Delta\left(p_{+}, n\right)$, следует, что сушествует $x \in\left(x_{0}, x_{0}+\delta\right)$ такое, что

$$
\left(Q(x)-f(x)+\alpha\left|x-x_{0}\right|^{n}\right) p_{+}(x) \geqslant|Q-f|_{p},
$$

поэтому для такого $x$ имеем

$$
\begin{aligned}
|P-f|_{p} & \geqslant(P(x)-f(x)) p_{+}(x)=(Q(x)-f(x)+P(x)-Q(x)) p_{+}(x) \\
& \geqslant\left(Q(x)-f(x)+\alpha\left|x-x_{0}\right|^{n}\right) p_{+}(x) \geqslant|Q-f|_{p},
\end{aligned}
$$

что противоречит (6), а поэтому при $x_{0} \in \Delta\left(p_{-}\right) \cap \Delta\left(p_{+}, n\right)$ (относительно своих правых окрестностей) выполняется равенство (1).

Случай $x_{0} \in \Delta\left(p_{+}\right) \cap \Delta\left(p_{-}, n\right)$ рассматривается вполне аналогично.

Пусть теперь точка $x_{0} \in \Delta\left(p_{-}\right) \cap \Delta_{+}^{\infty}(Q=f)^{\prime}$ относительно ее правых окрестностей. Тогда, как показано вьше, из того, что $x_{0} \in \Delta\left(p_{-}\right)$относительно правых окрестностей, и неравенства (6) следует, что $Q(x)<P(x)$ при $x \in\left(x_{0}, x_{0}+\delta\right)$ с некоторым достаточно малым $\delta>0$.

Из того, что $x_{0} \in \Delta_{+}^{\infty}(Q=f)^{\prime}$ относительно правых окрестностей, вытекает, что сушествует точка $y_{0} \in\left(x_{0}, x_{0}+\delta\right) \cap \Delta_{+}^{\infty}(Q=f)$. Тогда $P\left(y_{0}\right)>Q\left(y_{0}\right)=f\left(y_{0}\right)$, а поэтому $P(x)>f(x)$ (в силу непрерывности) при $x \in\left(y_{0}-\delta, y_{0}+\delta\right)$ с некоторым $\delta>0$. Так как $y_{0} \in \Delta_{+}^{\infty}$, получим

$$
|P-f|_{p} \geqslant \sup \left\{(P(x)-f(x)) p_{+}(x): x \in\left(y_{0}-\delta, y_{0}+\delta\right)\right\}=+\infty,
$$

что противоречит неравенству (6).

Случай $x_{0} \in \Delta\left(p_{+}\right) \cap \Delta_{-}^{\infty}(Q=f)^{\prime}$ (относительно правых окрестностей) рассматривается аналогично.

Пусть, далее, точка $x_{0} \in \Delta\left(p_{-}, n\right) \cap \Delta\left(p_{+}, n\right)$ относительно ее правых окрестностей. Возьмем $0<\alpha<(1 / 2)\left|a_{m}\right|$ для $a_{m} \neq 0,1 \leqslant m \leqslant n$,

$$
P(x)-Q(x)=a_{m}\left(x-x_{0}\right)^{m}+a_{m+1}\left(x-x_{0}\right)^{m+1}+\cdots+a_{n}\left(x-x_{0}\right)^{n},
$$

и $\delta>0$ такое, что разность $P(x)-Q(x)$ имеет определенный знак и вьполняется неравенство

$$
|P(x)-Q(x)| \geqslant \alpha\left|x-x_{0}\right|^{n}
$$

при $x \in\left(x_{0}, x_{0}+\delta\right)$. Тогда по определению $\Delta\left(p_{-}, n\right)$ и $\Delta\left(p_{+}, n\right)$ найдутся две последовательности точек $x_{j}, y_{j} \in\left(x_{0}, x_{0}+\delta\right)$ с $x_{j} \rightarrow x_{0}, y_{j} \rightarrow x_{0}, j \rightarrow \infty$ и

$$
\begin{aligned}
\left(f\left(x_{j}\right)-Q\left(x_{j}\right)+\alpha\left|x_{j}-x_{0}\right|^{n}\right) p_{-}\left(x_{j}\right) & \geqslant|Q-f|_{p}, \\
\left(Q\left(y_{j}\right)-f\left(y_{j}\right)+\alpha\left|y_{j}-x_{0}\right|^{n}\right) p_{+}\left(y_{j}\right) & \geqslant|Q-f|_{p} .
\end{aligned}
$$

Воспользуемся теперь первым или вторым из этих неравенств в зависимости от того, выполняется неравенство $Q(x)>P(x)$ или неравенство $Q(x)<P(x)$ при $x \in\left(x_{0}, x_{0}+\delta\right)$. Пусть, например, $Q(x)>P(x)$ при $x \in\left(x_{0}, x_{0}+\delta\right)$. Тогда

$$
\begin{aligned}
|P-f|_{p} & \geqslant\left(f\left(x_{j}\right)-P\left(x_{j}\right)\right) p_{-}\left(x_{j}\right)=\left(f\left(x_{j}\right)-Q\left(x_{j}\right)+Q\left(x_{j}\right)-P\left(x_{j}\right)\right) p_{-}\left(x_{j}\right) \\
& \geqslant\left(f\left(x_{j}\right)-Q\left(x_{j}\right)+\alpha\left|x_{j}-x_{0}\right|^{n}\right) p_{-}\left(x_{j}\right) \geqslant|Q-f|_{p}
\end{aligned}
$$

что противоречит неравенству (6). 
Рассмотрим теперь случай точки $x_{0} \in \Delta\left(p_{-}, n\right) \cap \Delta_{+}^{\infty}(Q=f)^{\prime}$ относительно ее правых окрестностей. Если при $x_{0} \in \Delta\left(p_{-}, n\right)$ допустить, что выполняется (6) и $Q(x)>P(x)$ для всех $x \in\left(x_{0}, x_{0}+\delta\right)$ с некоторьм $\delta>0$, то, как показано выше, приходим к противоречию. Поэтому пусть $Q(x)<P(x)$ для всех $x \in\left(x_{0}, x_{0}+\delta\right)$ с некоторым $\delta>0$.

Так как точка $x_{0} \in \Delta_{+}^{\infty}(Q=f)^{\prime}$ относительно ее правых окрестностей, то сушествует $y_{0} \in\left(x_{0}, x_{0}+\delta\right) \cap \Delta_{+}^{\infty}(Q=f)$. Тогда $P\left(y_{0}\right)>Q\left(y_{0}\right)=f\left(y_{0}\right)$ и в силу непрерывности $P(x)>f(x)$ при всех $x \in\left(y_{0}-\eta, y_{0}+\eta\right)$ с некоторым $\eta>0$. Отсюда и из $y_{0} \in \Delta_{+}^{\infty}$, как и вьше, приходим к противоречию: $|P-f|_{p}=\infty$.

Случай $x_{0} \in \Delta\left(p_{+}, n\right) \cap \Delta_{-}^{\infty}(Q=f)^{\prime}$ относительно правых окрестностей рассматривается аналогично.

Пусть, наконец, точка $x_{0} \in \Delta_{-}^{\infty}(Q=f)^{\prime} \cap \Delta_{+}^{\infty}(Q=f)^{\prime}$ относительно ее правых окрестностей. Тогда ввиду конечности $|P-f|_{p}$ найдутся такие две последовательности различных точек $x_{j}$ и различных точек $y_{j}$, что

$$
\begin{aligned}
& x_{j}>x_{0}, \quad x_{j} \in \Delta_{-}^{\infty}, \quad Q\left(x_{j}\right)=f\left(x_{j}\right), \quad f\left(x_{j}\right)-P\left(x_{j}\right) \leqslant 0, \\
& y_{j}>x_{0}, \quad y_{j} \in \Delta_{+}^{\infty}, \quad Q\left(y_{j}\right)=f\left(y_{j}\right), \quad f\left(y_{j}\right)-P\left(y_{j}\right) \geqslant 0 .
\end{aligned}
$$

Следовательно, $Q\left(x_{j}\right) \leqslant P\left(x_{j}\right)$ и $Q\left(y_{j}\right) \geqslant P\left(y_{j}\right)$ для всех таких различных точек $x_{j}$ и $y_{j}$, а поэтому $Q(x) \equiv P(x)$, что противоречит неравенству (6).

Итак, в условиях теоремы вьполняется равенство (1) и теорема 2 доказана.

\section{§3. Точки $p$-экстремума полной степени. Второе достаточное условие полинома наилучшего приближения}

Пусть, как и выше, вес $p=\left(p_{-}, p_{+}\right)$определен на отрезке $\Delta$, функция $f(x)$ непрерывна на $\Delta, Q(x)$ - заданньй алгебраический полином степени не вьше $n$, $n=0,1, \ldots$, причем $Q(x) \geqslant f(x)$ при $x \in \Delta_{-}^{\infty}$ и $Q(x) \leqslant f(x)$ при $x \in \Delta_{+}^{\infty}$.

При определении точки $p$-экстремума полной степени будем считать, что на отрезке $\Delta$ нет точек, принадлежащих множеству $E_{-} \cap E_{+}$относительно своих односторонних окрестностей. Тогда, в частности, $|Q-f|_{p}>0$.

Если точка $z \notin E_{-}$относительно ее правых окрестностей, то через $\nu_{r-}(z)$ обозначим наименьшее натуральное $\nu \leqslant n$, для которого существуют сколь угодно малые $\alpha>0$ и $\delta>0$ такие, что

$$
\sup \left\{\left(f(x)-Q(x)+\alpha|x-z|^{\nu}\right) p_{-}(x): x \in[z, z+\delta]\right\}<|Q-f|_{p} .
$$

Заметим, что величина $\nu_{r-}(z)$ существует, так как в противном случае получили бы $z \in \Delta\left(p_{-}\right)$или $z \in \Delta\left(p_{-}, n\right)$, а поэтому $z \in E_{-}$относительно правых окрестностей точки $z$.

Как легко видеть, при $\nu=\nu_{r-}(z)$, натуральных $m<\nu$ и сколь угодно малом $\alpha>0$ найдется такая последовательность точек $x_{k} \rightarrow 0, k \rightarrow \infty, x_{k} \geqslant z$, что

$$
\left(f\left(x_{k}\right)-Q\left(x_{k}\right)+\alpha\left|x_{k}-z\right|^{m}\right) p_{-}\left(x_{k}\right) \geqslant|Q-f|_{p}
$$

Взяв в (7) левую окрестность $[z-\delta, z]$ вместо правой $[z, z+\delta]$, получим определение величины $\nu_{l-}(z)$. 
Вполне аналогично относительно другой компоненты веса $p_{+}(x)$ определяются величины $\nu_{r+}(z)$ и $\nu_{l+}(z)$, при этом условие $(7)$ следует заменить на условие

$$
\sup \left(Q(x)-f(x)+\alpha|x-z|^{\nu}\right) p_{+}(x)<|Q-f|_{p},
$$

где супремум берется соответственно на $[z, z+\delta]$ и на $[z-\delta, z]$.

Для точек $p$-экстремума $z \in \Delta\left(p_{-}\right) \cup \Delta\left(p_{+}\right)$определим также другую характеристику; именно, если точка $z \in \Delta\left(p_{+}\right)$относительно ее правых окрестностей, то $\mu_{r+}(z)$ означает наименьшее натуральное число $\mu \leqslant n$, для которого найдется последовательность точек $y_{k} \rightarrow z, k \rightarrow \infty, y_{k} \geqslant z$, такая, что

$$
\left(y_{k}-z\right)^{\mu} p_{+}\left(y_{k}\right) \rightarrow 0, \quad\left(Q\left(y_{k}\right)-f\left(y_{k}\right)\right) p_{+}\left(y_{k}\right) \rightarrow|Q-f|_{p}
$$

при $k \rightarrow \infty$; в противном случае для этой точки $z \in \Delta\left(p_{+}\right)$положим $\mu_{r+}(z)=$ $n+1$.

Заметим, что если $\mu_{r+}(z)=n+1$, то при любом $1 \leqslant \mu \leqslant n$ для любой последовательности $y_{k} \rightarrow z, k \rightarrow \infty, y_{k} \geqslant z$, такой, что

$$
\left(Q\left(y_{k}\right)-f\left(y_{k}\right)\right) p_{+}\left(y_{k}\right) \rightarrow|Q-f|_{p}
$$

величина $\left(y_{k}-z\right)^{\mu} p_{+}\left(y_{k}\right)$ не стремится к нулю при $k \rightarrow \infty$.

Взяв в (10) последовательность $y_{k} \leqslant z$ вместо $y_{k} \geqslant z$, получим определение величины $\mu_{l+}(z)$ относительно левых окрестностей точки $z$.

Аналогично определяются $\mu_{r-}(z)$ и $\mu_{l-}(z)$ для компоненты $p_{-}(x)$, если точка $z \in \Delta\left(p_{-}\right)$.

Рассмотрим теперь полиномы вида

$$
P_{m, n}(x)=Q(x)+a_{m}(x-z)^{m}+\cdots+a_{n}(x-z)^{n}
$$

с заданным полиномом $Q(x)$ и действительнымикоэффициентами $a_{m}, a_{m+1}, \ldots, a_{n}$, $1 \leqslant m \leqslant n$.

Тогда для рассматриваемых на отрезке $\Delta$ веса $p=\left(p_{-}, p_{+}\right)$, непрерывной функции $f(x)$ и полинома $Q(x)$ степени $\leqslant n, n=0,1, \ldots$, с $Q(x) \geqslant f(x)$ при $x \in \Delta_{-}^{\infty}$ и $Q(x) \leqslant f(x)$ при $x \in \Delta_{+}^{\infty}$ имеют место следующие утверждения.

Лемма 1. Если существует точка $z \in F=\Delta_{-}^{\infty} \cap \Delta_{+}^{\infty}$, то при $n=0$ для любого полинома $P(x)$ нулевой степени $|Q-f|_{p} \leqslant|P-f|_{p}$, а при $n \geqslant 1$ для некоторого полинома $P(x)$ степени не выше $n$ неравенство

$$
|P-f|_{p}<|Q-f|_{p}
$$

возмодно лишь при его совпадении с одним из полиномов вида $P_{m, n}(x)$, $1 \leqslant m \leqslant n$.

Отметим, что в случае $z \in \Delta\left(p_{-}\right) \cup \Delta\left(p_{+}\right)$и $n \geqslant 1$ значение параметра $m$, при котором полином $P(x)=P_{m, n}(x)$ может удовлетворять неравенству (11), можно дальше уточнить. Лемму 1 дополняет 
Лемма 2. При $n \geqslant 1$ и z $\in F=\Delta_{-}^{\infty} \cap \Delta_{+}^{\infty}$ неравенство

$$
\left|P_{m, n}-f\right|_{p, \Delta}<|Q-f|_{p, \Delta}
$$

возможно лишь при значении $m$, удовлетворяющем двойному неравенству

$$
\nu_{r-}(z) \leqslant m<\mu_{r+}(z) \quad\left(\nu_{l-}(z) \leqslant m<\mu_{l+}(z)\right)
$$

для точки $z \in \Delta\left(p_{+}\right) \subset E_{+}$относительно ее правых (левых) окрестностей и двойному неравенству

$$
\nu_{r+}(z) \leqslant m<\mu_{r-}(z) \quad\left(\nu_{l+}(z) \leqslant m<\mu_{l-}(z)\right)
$$

для точки $z \in \Delta\left(p_{-}\right) \subset E_{-}$относительно ее правых (левых) окрестностей. При этом число $m$ четное или нечетное в зависимости от того, точка $z$ принадлежит одному и тому же или разным из двух множсеств $E_{-} u E_{+}$ относительно ее левых и правых окрестностей.

Приведенные в лемме 2 двойные неравенства для краткости всюду ниже назовем согласованньми с точкой $z \in \Delta\left(p_{-}\right) \cup \Delta\left(p_{+}\right)$относительно ее соответствующих односторонних окрестностей и определим точки $p$-экстремума полной степени.

Пусть на отрезке $\Delta=[a, b]$ заданы произвольньй вес $p=\left(p_{-}, p_{+}\right)$и непрерывная функция $f(x) ; Q \in \mathscr{P}_{n}, n=0,1, \ldots$, причем $Q(x) \geqslant f(x)$ при $x \in \Delta_{-}^{\infty}$ и $Q(x) \leqslant f(x)$ при $x \in \Delta_{+}^{\infty}$; точка $z \in \Delta_{-}^{\infty} \cap \Delta_{+}^{\infty}$, но эта точка $z \notin E_{-} \cap E_{+}$ относительно одних и тех же ее односторонних окрестностей.

Через $m(z)$ обозначим натуральное число, четное или нечетное в зависимости от того, точка $z$ принадлежит одному и тому же или разным из двух множеств $E_{-}$ и $E_{+}$относительно ее правых и левых окрестностей, и произвольное в остальных случаях.

ОПРЕДЕЛЕНИЕ 1. Точку $z \in \Delta\left(p_{-}\right) \cup \Delta\left(p_{+}\right)$назовем точкой $p$-экстремума полной степени для разности $Q(x)-f(x)$ на отрезке $\Delta$, если $n=0$ или если $n \geqslant 1$ и хотя бы одно согласованное с этой точкой $z$ двойное неравенство среди следующих четырех неравенств

$$
\begin{gathered}
\nu_{r \mp}(z) \leqslant m<\mu_{r \pm}(z), \\
\nu_{l \mp}(z) \leqslant m<\mu_{l \pm}(z)
\end{gathered}
$$

не имеет решения типа $m=m(z)$.

Из данного определения 1 и лемм 1 и 2 непосредственно вытекает следующая

ТЕОРема 3. Если для заданных на отрезке $\Delta$ веса $p=\left(p_{-}, p_{+}\right)$, непрерывной функции $f(x)$ и полинома $Q \in \mathscr{P}_{n}, n=0,1, \ldots$, существует хотя бъ одна точка р-экстремума полной степени для разности $Q(x)-f(x)$ на $\Delta$, то $Q(x)$ - полином наилучшего приближения порядка $n$ функции $f(x)$ в р-норме с весом $p=\left(p_{-}, p_{+}\right)$на отрезке $\Delta$. 
ДОКАЗАТЕЛЬСТво ЛЕМмы 1 . Из $z \in \Delta_{-}^{\infty} \cap \Delta_{+}^{\infty}$ по условию на полином $Q(x)$ и функцию $f(x)$ имеем $Q(z)=f(z)$.

Поэтому при $n=0$ получим: $|Q-f|_{p}=|P-f|_{p}$, если полиномы $Q(x)$ и $P(x)$ совпадают, и $|P-f|_{p}=\infty$, если эти полиномы не совпадают.

При $n \geqslant 1$ из неравенства (11) следует конечность величины $|P-f|_{p}$, поэтому $P(z)=f(z)$, так как в силу непрерьвности $P(x)-f(x)$ при $P(z)>f(z)$ и $P(z)<$ $f(z)$ получим $|P-f|_{p}=\infty$. Так как $P(z)=f(z)=Q(z)$, то второе утверждение леммы также доказано.

ДоКАЗАТЕЛЬСтво ЛЕммы 2. Сначала покажем, что если $\mu$ совпадает с одной из четырех величин $\mu_{r \pm}(z), \mu_{l \pm}(z)$ и $\mu \leqslant m \leqslant n$, то

$$
|Q-f|_{p} \leqslant\left|P_{m, n}-f\right|_{p}
$$

Действительно, пусть для определенности сушествует $\mu=\mu_{r+}(z) \leqslant m \leqslant n$. Тогда для последовательности точек $y_{k}$ из определения величины $\mu_{r+}(z)$ при $k \rightarrow \infty$ получим

$$
\begin{aligned}
\left|P_{m, n}-f\right|_{p} \geqslant & \left(P_{m, n}\left(y_{k}\right)-f\left(y_{k}\right)\right) p_{+}\left(y_{k}\right)=\left(Q\left(y_{k}\right)-f\left(y_{k}\right)\right) p_{+}\left(y_{k}\right) \\
& +\left(a_{m}\left(y_{k}-z\right)^{m}+\cdots+a_{n}\left(y_{k}-z\right)^{n}\right) p_{+}\left(y_{k}\right) \rightarrow|Q-f|_{p} .
\end{aligned}
$$

Следовательно, если $z \in F, \mu$ равно одной из величин $\mu_{r \pm}(z), \mu_{l \pm}(z)$ и для некоторого полинома $P(x)$ степени не выше $n$ выполняется неравенство (11), то $P(x) \equiv P_{m, n}(x)$ при некотором $m<\mu$.

Далее, если неравенство (11) выполняется для некоторого полинома вида $P(x)=$ $P_{m, n}(x)$, то для $x$ из некоторой правой или левой окрестности точки $z$ имеем $a_{m}(x-z)^{m}<0$ при $z \in \Delta\left(p_{+}\right)$(аналогично, $a_{m}(x-z)^{m}>0$ при $z \in \Delta\left(p_{-}\right)$) относительно (соответственно) правой или левой окрестности точки $z$.

В самом деле, если $z \in \Delta\left(p_{+}\right)$и $z \notin \Delta_{+}^{\infty}$, то

$$
(Q(z)-f(z)) p_{+}(z)=|Q-f|_{p}>(P(z)-f(z)) p_{+}(z),
$$

поэтому $Q(z)>P(z)$ и в силу непрерывности полиномов имеем

$$
P(x)-Q(x)=a_{m}(x-z)^{m}+\cdots+a_{n}(x-z)^{n}<0,
$$

откуда $a_{m}(x-z)^{m}<0$ в некоторой окрестности точки $z$ при $x \neq z$.

Если точка $z \in \Delta_{+}^{\infty} \cap \Delta\left(p_{+}\right)$относительно, например, правых ее окрестностей, то найдется последовательность точек $y_{k} \rightarrow z, y_{k}>z, \mathrm{c}\left(Q\left(y_{k}\right)-f\left(y_{k}\right)\right) p_{+}\left(y_{k}\right) \rightarrow$ $|Q-f|_{p}$ при $k \rightarrow \infty$. Тогда из неравенства (11) получим

$$
\left(Q\left(y_{k}\right)-f\left(y_{k}\right)\right) p_{+}\left(y_{k}\right)>\left(P\left(y_{k}\right)-f\left(y_{k}\right)\right) p_{+}\left(y_{k}\right)
$$

(начиная с некоторого номера $k$ ), а поэтому $Q\left(y_{k}\right)>P\left(y_{k}\right)$ или $Q(x)>P(x)$ при $x \in(z, z+\delta)$ с некоторьм $\delta>0$. 
Отсюда и из $P(x)=P_{m, n}(x)$, как и выше, получим $a_{m}(x-z)^{m}<0$ при $x \in$ $(z, z+\delta)$ с некоторьм $\delta>0$.

Случаи $z \in \Delta\left(p_{-}\right) \backslash \Delta_{-}^{\infty}$ и $z \in \Delta_{-}^{\infty} \cap \Delta\left(p_{-}\right)$рассматриваются аналогично.

Пусть теперь $z \in \Delta\left(p_{-}\right) \cup \Delta\left(p_{+}\right)$, причем точка $z$ принадлежит одному и тому же из множеств $E_{+}$и $E_{-}$относительно обеих своих односторонних окрестностей.

Если при этом выполняется неравенство (11) с $P(x)=P_{m, n}(x)$, то число $m$ четное.

Действительно, если, например, точка $z \in \Delta\left(p_{+}\right)$относительно ее правых окрестностей, то, как показано, из неравенства (11) следует, что $P(x)=P_{m, n}(x)<$ $Q(x)$ при $x \in(z, z+\delta)$ с некоторым $\delta>0$.

Если теперь точка $z \in E_{+}$, причем относительно ее левых окрестностей, то относительно своих левых окрестностей точка $z$ принадлежит одному из следующих трех множеств: $\Delta\left(p_{+}\right), \Delta\left(p_{+}, n\right)$ или $\Delta_{+}^{\infty}(Q=f)^{\prime}$.

Если $z \in \Delta\left(p_{+}\right)$относительно левых окрестностей, то возьмем последовательность точек $y_{k} \rightarrow z, y_{k} \leqslant z$, такую, что

$$
\left(Q\left(y_{k}\right)-f\left(y_{k}\right)\right) p_{+}\left(y_{k}\right) \rightarrow|Q-f|_{p}>0
$$

при $k \rightarrow \infty$. Тогда, как и выше, $P\left(y_{k}\right)<Q\left(y_{k}\right)$ для некоторой подпоследовательности $y_{k_{i}}$, а поэтому $P(x)<Q(x)$ при $x \in(z-\delta, z)$ с некоторым $\delta>0$.

Следовательно, в некоторой проколотой $\delta$-окрестности точки $z$ выполняется неравенство

$$
P(x)-Q(x)=a_{m}(x-z)^{m}+\cdots+a_{n}(x-z)^{n}<0,
$$

а поэтому $m$ четное (так как $P(z)=Q(z)$ и $m \neq 0$ ).

Если точка $z \in \Delta\left(p_{+}, n\right)$ относительно ее левых окрестностей, то по определению $\Delta\left(p_{+}, n\right)$ при $0<\alpha<(1 / 4)\left|a_{m}\right|$ найдется последовательность точек $y_{k} \rightarrow z$, $y_{k} \leqslant z$, такая, что

$$
\left(Q\left(y_{k}\right)-f\left(y_{k}\right)+\alpha\left|y_{k}-z\right|^{n}\right) p_{+}\left(y_{k}\right) \geqslant|Q-f|_{p} .
$$

Допустим теперь от противного, что при $x \in(z-\delta, z)$ с некоторым $\delta>0$ имеем, что $P(x)>Q(x)$ и выполняется неравенство

$$
P(x)-Q(x)=a_{m}(x-z)^{m}+\cdots+a_{n}(x-z)^{n}>\frac{1}{2}\left|a_{m}\right||x-z|^{m} .
$$

Тогда для всех тех $y_{k}$, которые принадлежат интервалу $(z-\delta, z)$, получим

$$
\begin{aligned}
|P-f|_{p} & \geqslant\left(P\left(y_{k}\right)-f\left(y_{k}\right)\right) p_{+}\left(y_{k}\right)=\left(Q\left(y_{k}\right)-f\left(y_{k}\right)+P\left(y_{k}\right)-Q\left(y_{k}\right)\right) p_{+}\left(y_{k}\right) \\
& \geqslant\left(Q\left(y_{k}\right)-f\left(y_{k}\right)+\frac{1}{2}\left|a_{m}\right|\left|y_{k}-z\right|^{m}\right) p_{+}\left(y_{k}\right) \geqslant|Q-f|_{p},
\end{aligned}
$$

что противоречит неравенству (11).

Следовательно, если $z \in \Delta\left(p_{+}, n\right)$ относительно левых окрестностей, то также выполняется условие (12) и число $m$ четное. 
Если, наконец, точка $z \in \Delta_{+}^{\infty}(Q=f)^{\prime}$ относительно ее левых окрестностей, то по определению этого множества найдется последовательность точек $y_{k} \rightarrow z$, $y_{k} \leqslant z$, с $Q\left(y_{k}\right)=f\left(y_{k}\right)$ и $y_{k} \in \Delta_{+}^{\infty}$.

Если теперь допустить, что $P(x)>Q(x)$ при $x \in(z-\delta, z)$ с некоторым $\delta>0$, то найдется $y_{k} \in(z-\delta, z), P\left(y_{k}\right)>Q\left(y_{k}\right)=f\left(y_{k}\right)$, причем сушествует последовательность точек $y_{k, i} \rightarrow y_{k}, p_{+}\left(y_{k, i}\right) \rightarrow+\infty$ при $i \rightarrow \infty$. Тогда, как легко видеть,

$$
|P-f|_{p} \geqslant\left(P\left(y_{k, i}\right)-f\left(y_{k, i}\right)\right) p_{+}\left(y_{k, i}\right)=\left(P\left(y_{k}\right)-Q\left(y_{k}\right)+\varphi\left(y_{k, i}\right)\right) p_{+}\left(y_{k, i}\right)
$$

с некоторой последовательностью $\varphi\left(y_{k, i}\right) \rightarrow 0$ при $i \rightarrow \infty$ и фиксированньм значением $P\left(y_{k}\right)-Q\left(y_{k}\right)>0$, а поэтому $|P-f|_{p}=\infty$, что противоречит неравенству (11).

Следовательно, выполняется (12) и число $m$ четное.

Вполне аналогично рассматривается случай $z \in \Delta\left(p_{-}\right)$, когда $z \in E_{-}$относительно обеих односторонних окрестностей точки $z$; в этом случае число $m$ также является четным.

Если же $z \in \Delta\left(p_{-}\right) \cup \Delta\left(p_{+}\right)$, причем точка $z$ принадлежит разньм из двух множеств $E_{+}$и $E_{-}$относительно левых и правых окрестностей этой точки, и выполняется неравенство (11) с $P(x)=P_{m, n}(x)$, то, аналогично, число $m$ нечетное.

Пусть теперь данная точка $z \in \Delta\left(p_{-}\right) \cup \Delta\left(p_{+}\right)$принадлежит также множеству $\Delta_{-}^{\infty} \cap \Delta_{+}^{\infty}$, значение $\nu$ совпадает с одной из четырех величин $\nu_{r \pm}(z), \nu_{l \pm}(z)$. Выясним, при каких условиях на $\nu$ не сушествует полинома $P(x)$ степени не выше $n$, удовлетворяющего неравенству (11).

Если (11) выполнено для полинома $P(x)$ и точка $z \in \Delta_{-}^{\infty} \cap \Delta_{+}^{\infty}$, то, как уже показано, $P(x)$ совпадает с полиномом вида $P_{m, n}(x)$ при некотором натуральном $m \leqslant n$ и некоторых действительных значениях коэффициентов $a_{m}, a_{m+1}, \ldots, a_{n}$.

Пусть для определенности $\nu=\nu_{r-}(z)$ и точка $z \in \Delta\left(p_{+}\right)$относительно ее правых окрестностей. Допустим, что указанное $m<\nu$.

Тогда из выполнения неравенства (11) вытекает, что $m<\mu_{r+}(z)$ и произведение $a_{m}(x-z)^{m}<0$ при $x \in(z, z+\delta)$ с некоторым $\delta>0$.

По определению величины $\nu_{r-}(z)$ существует такая последовательность точек $x_{k} \rightarrow z, x_{k} \geqslant z$, что выполняется (8). Тогда при достаточно больших номерах $k$ и $0<\alpha<(1 / 2)\left|a_{m}\right|$ получим

$$
\begin{aligned}
\left(f\left(x_{k}\right)\right. & \left.-P\left(x_{k}\right)\right) p_{-}\left(x_{k}\right) \\
& =\left(f\left(x_{k}\right)-Q\left(x_{k}\right)-a_{m}\left(x_{k}-z\right)^{m}-\cdots-a_{n}\left(x_{k}-z\right)^{n}\right) p_{-}\left(x_{k}\right) \\
& \geqslant\left(f\left(x_{k}\right)-Q\left(x_{k}\right)+\frac{1}{2}\left|a_{m}\right|\left|x_{k}-z\right|^{m}\right) p_{-}\left(x_{k}\right) \geqslant|Q-f|_{p},
\end{aligned}
$$

что противоречит (11).

Итак, когда $z \in \Delta_{-}^{\infty} \cap \Delta_{+}^{\infty}$ и эта точка $z \in \Delta\left(p_{+}\right) \subset E_{+}$относительно ее правых окрестностей, полином $P(x)$ степени не выше $n, n \geqslant 1, \mathrm{c}|P-f|_{p}<|Q-f|_{p}$ может сушествовать, если только $P(x)=P_{m, n}(x)$ со значением $m$, удовлетворяющим двойному неравенству

$$
\nu_{r-}(z) \leqslant m<\mu_{r+}(z)
$$


причем $m$ четное, если дополнительно точка $z \in E_{+}$относительно ее левых окрестностей, и $m$ нечетное, если относительно нее $z \in E_{-}$, т.е. удовлетворяющим двойному неравенству (14), согласованному с точкой $z \in \Delta\left(p_{+}\right)$относительно ее правых окрестностей.

Вполне аналогичные утверждения справедливы для остальных возможных случаев точки $z \in \Delta\left(p_{+}\right)$и точки $z \in \Delta\left(p_{-}\right)$, причем для них согласованными будут следуюшие двойные неравенства:

$$
\nu_{l-}(z) \leqslant m<\mu_{l+}(z)
$$

- для $z \in \Delta\left(p_{+}\right)$относительно ее левых окрестностей;

$$
\nu_{r+}(z) \leqslant m<\mu_{r-}(z) \text { и } \nu_{l+}(z) \leqslant m<\mu_{l-}(z)
$$

- для $z \in \Delta\left(p_{-}\right)$относительно ее правых и левых окрестностей соответственно.

Лемма 2 доказана.

\section{§4. Полиномы оптимального перехода. Индекс альтернанса}

Пусть на отрезке $\Delta=[a, b]$ заданы произвольньй вес $p=\left(p_{-}, p_{+}\right)$и непрерывная функция $f(x)$; полином $Q \in \mathscr{P}_{n}, n=0,1, \ldots ;$ мощность множества $F=\Delta_{-}^{\infty} \cap \Delta_{+}^{\infty}$ равна $k \leqslant n ; Q(x) \geqslant f(x)$ при $x \in \Delta_{-}^{\infty}$ и $Q(x) \leqslant f(x)$ при $x \in \Delta_{+}^{\infty}$.

При определении индекса альтернанса будем считать также, что множество $E_{-} \cap E_{+}$не содержит точек $p$-экстремума полной степени для разности $Q(x)-f(x)$ на $\Delta$ и точек, принадлежащих множеству $E_{-} \cap E_{+}$относительно их одних и тех же односторонних окрестностей; тогда, в частности, $|Q-f|_{p}>0$ и при $n=0$ на $\Delta$ нет точек множества $F$ (т.е. $k=0)$.

Пусть, далее, $z_{1}<z_{2}<\cdots<z_{k}$ суть все точки множества $F, z_{0}=a, z_{k+1}=b$; $z_{0} \leqslant z_{1}, z_{k} \leqslant z_{k+1}$. Тогда на каждом из промежутков

$$
\left[z_{0}, z_{1}\right), \quad\left(z_{1}, z_{2}\right), \ldots, \quad\left(z_{k}, z_{k+1}\right]
$$

при $F \neq \varnothing$ (и на отрезке $\left[z_{0}, z_{k+1}\right]$ при $\left.F=\varnothing\right)$ найдется самое большее конечное число $m_{i}, i=0,1, \ldots, k$, точек

$$
t_{1}^{(i)}<t_{2}^{(i)}<\cdots<t_{m_{i}}^{(i)}
$$

таких, что они не принадлежат множеству $G_{-} \cup G_{+}$, а интервалы

$$
\left(z_{i}, t_{1}^{(i)}\right), \quad\left(t_{1}^{(i)}, t_{2}^{(i)}\right), \ldots, \quad\left(t_{m_{i}}^{(i)}, z_{i+1}\right)
$$

пересекаются то с множеством $G_{-}$(их назовем $(-)$-интервалами), то с множеством $G_{+}$(их назовем $(+)$-интервалами) с чередованием, причем для каждого промежутка в отдельности, начиная с любого из этих двух множеств; полагаем $m_{i}=0$, когда соответствующий промежуток пересекается не более чем с одним из множеств $G_{-}$и $G_{+}$. 
Для каждой из точек $z=z_{i}, i=1,2, \ldots, k$, определим теперь полином $P(x, z)$ оптимального перехода через точку $z$. Заметим, что речь идет о полиномах, имеющих нули в точках $z \in F$, в любой окрестности которых не ограничены обе компоненты веса; поэтому определяются они в случае полинома $Q \in \mathscr{P}_{n}$ с $n \geqslant 1$ (так как у нас $F=\varnothing$ при $n=0)$.

Пусть $z \in F$. Тогда, как следует из определения величин $\nu_{r \pm}(z)$ и $\nu_{l \pm}(z)$, если точка $z \in E_{+}\left(z \in E_{-}\right)$относительно ее правых или левых окрестностей, то существует $\nu_{r-}(z)$ или $\nu_{l-}(z)\left(\nu_{r+}(z)\right.$ или $\left.\nu_{l+}(z)\right)$.

Действительно, в случае $z \in E_{+}\left(z \in E_{-}\right)$относительно данных односторонних окрестностей имеем $z \notin E_{-}$(соответственно $\left.z \notin E_{+}\right)$относительно тех же односторонних окрестностей, а поэтому указанные величины существуют (у нас $z \notin E_{-} \cap E_{+}$относительно одних и тех же односторонних окрестностей).

Точнее, для данњых правых (соответственно левых) окрестностей точки $z \in F$ имеем: если $z \in E_{+} \backslash E_{-}$, то сушествует $\nu_{r-}(z)\left(\nu_{l-}(z)\right)$; если $z \in E_{-} \backslash E_{+}$, то существует $\nu_{r+}(z)\left(\nu_{l+}(z)\right)$; если, наконец, $z \notin E_{-} \cup E_{+}$, то существуют обе величины $\nu_{r \pm}(z)\left(\nu_{l \pm}(z)\right)$ (случай $z \in E_{-} \cap E_{+}$относительно односторонних окрестностей исключен; точки $p$-экстремума полной степени также исключены).

Чтобы определить степень полинома $P(x, z)$ оптимального перехода, для данной точки $z \in F$ возьмем целые решения $m=m(z)$ соответствующих точке $z$ систем неравенств вида

$$
\left\{\begin{array}{l}
\nu_{r \mp}(z) \leqslant m<\mu_{r \pm}(z), \\
\nu_{l \mp}(z) \leqslant m<\mu_{ \pm}(z),
\end{array}\right.
$$

точнее, систем с допустимыми (существующими) для данной точки $z$ левьми частями неравенств; причем правая часть неравенства опускается в случае ее несуществования, т.е. при $z \notin \Delta\left(p_{-}\right) \cup \Delta\left(p_{+}\right)$, а в случае существования правой части берется двойное неравенство, согласованное с данной точкой $z$ относительно ее соответствующих односторонних окрестностей.

Пусть, например, точка $z \in E_{+} \backslash E_{-}$относительно ее правых окрестностей. Тогда в качестве первого неравенства соответствующей этой точке системы берем $\nu_{r-}(z) \leqslant m<\mu_{r+}(z)$, а второе неравенство выбираем в зависимости от того, какому множеству принадлежит точка $z: z \in E_{-} \backslash E_{+}, z \in E_{+} \backslash E_{-}$или $z \notin$ $E_{-} \cup E_{+}$относительно своих левых окрестностей. Получим соответственно три групшы систем неравенств: одну систему с неравенством $\nu_{l+}(z) \leqslant m<\mu_{l-}(z)$ в качестве второго, другую - с неравенством $\nu_{l-}(z) \leqslant m<\mu_{l+}(z)$ в качестве второго и две системы с неравенствами $\nu_{l+}(z) \leqslant m$ и $\nu_{l-}(z) \leqslant m$ в качестве вторых.

Аналогично, по три группы соответствующих систем неравенств получим для точки $z \in E_{-} \backslash E_{+}$относительно ее правых и ее левых окрестностей и три группы систем для точки $z \in E_{+} \backslash E_{-}$относительно ее левых окрестностей.

В случае, когда точка $z \notin E_{-} \cup E_{+}$относительно обеих своих односторонних окрестностей, получим одну группу из четырех соответствующих этой точке систем, образованных всеми парами разных неравенств из $\nu_{r \pm}(z) \leqslant m, \nu_{l \pm}(z) \leqslant m$.

Возьмем теперь группу соответствующих данной точке $z \in F$ систем неравенств рассматриваемого вида и найдем наименьшее целое решение $m=m(z)$ отдельно для каждой системы неравенств этой групшы. При этом $m(z)$ обозначает чис- 
ло четное или нечетное в тех случаях, когда точка $z$ принадлежит одному и тому же или, соответственно, разным из двух множеств $E_{-}$и $E_{+}$относительно ее правых и левых окрестностей, а также в тех случаях, когда $z \notin E_{-} \cap E_{+}$, но является общим концом двух соответственно одноименных или разноименных $(+)-$ и (-)-интервалов.

$\mathrm{C}$ найденным $m=m(z)$ составим выражение $\pm(x-z)^{m}$, причем знак + или выбирается таким образом, чтобы в правой и, отдельно, в левой окрестности точки $z$ это выражение было положительно или отрицательно в зависимости от того, содержит данная система неравенство с величиной $\nu_{r+}(z)$ или с другой $\nu_{r-}(z)$ и соответственно с $\nu_{l+}(z)$ или с $\nu_{l-}(z)$; в случае точки $z \in E_{-} \cup E_{+}$это эквивалентно $z \in E_{-} \backslash E_{+}$или $z \in E_{+} \backslash E_{-}$относительно правых окрестностей и соответственно левых окрестностей точки $z$.

Пусть сначала точка $z \in F$ является общим концом двух каких-нибудь $(+)-$ и/или $(-)$-интервалов $(\alpha, z)$ и $(z, \beta)$. Если при этом $z \in E_{-} \cup E_{+}$относительно данных левых или правых окрестностей и на $(+)$-интервале (на $(-)$-интервале) $(\alpha, z)$ или $(z, \beta)$ выражение $\pm(x-z)^{m}$ (с уже выбранным знаком + или -) окажется положительньм (соответственно отрицательным), то возьмем дополнительно точку $z-\delta \in(\alpha, z)$ или $z+\delta \in(z, \beta)$ с достаточно малым $\delta>0$; в противном случае дополнительная точка $z-\delta$ или $z+\delta$ не берется. Тогда в зависимости от существования дополнительных точек получится один из следующих четырех полиномов:

$$
\begin{aligned}
& \pm(x-z)^{m}(x-z+\delta)(x-z-\delta), \quad \pm(x-z)^{m}, \\
& \pm(x-z)^{m}(x-z+\delta), \quad \pm(x-z)^{m}(x-z-\delta),
\end{aligned}
$$

степень $\gamma(z)$ которого равна одному из чисел $m(z)+2, m(z)$ или $m(z)+1$.

Если для данной точки $z$ группа соответствующих ей систем неравенств содержит более одной системы, то в этой группе выбираем ту систему неравенств, для которой степень $\gamma(z)$ получается наименьшей. Соответствующий полином $P(x, z)$ среди указанных четырех полиномов назовем полиномом оптимального перехода через точку z.

В случае, когда концы отрезка $\Delta=[a, b]$ принадлежат $F$ и являются концами $(+)$ - или (-)-интервалов, вместо системы неравенств берем соответствующее данному концу одно из этих неравенств (как и выше, в зависимости от того, какому из множеств $E_{-}$или $E_{+}$данный конец отрезка принадлежит или не принадлежит).

Заметим, что на отрезке $\Delta$ найдется хотя бы одна точка множества $\Delta\left(p_{-}\right) \cup$ $\Delta\left(p_{+}\right)$; она может совпадать с одной из точек $z_{i}, i=0,1, \ldots, k+1$, или принадлежать одному из интервалов $\left(z_{i}, z_{i+1}\right), i=0,1, \ldots, k$. На тех интервалах, которые пересекаются с $G_{-} \cup G_{+}$, построим $(+)$- и/или $(-)$-интервалы.

Рассмотрим теперь такие точки $z_{i}$ и $z_{j}$ (если существуют), что каждая из них в отдельности принадлежит $E_{-} \cup E_{+}$или $z_{i}\left(z_{j}\right)$ - правый (левый) конец некоторого $(+)$ - или $(-)$-интервала и на $\left(z_{i}, z_{j}\right)$ нет вообще $(+)$ - или $(-)$-интервалов; при этом возможны равенства $a=z_{i}$ и $b=z_{j}$.

Для каждой из точек $z=z_{i}, z_{i+1}, \ldots, z_{j}$ возьмем, как и выше, группу соответствующих систем неравенств, найдем минимальное целое решение $m=m(z)$ каждой системы и построим полиномы вида $\pm(x-z)^{m}$, причем при $z=z_{l}, l=$ $i, i+1, \ldots, j$, знак + или - перед $(x-z)^{m(z)}$ выбирается так, чтобы полученное 
после этого выражение было положительным (отрицательньм) в некоторой правой окрестности точки $z$, если $\nu_{r+}(z) \leqslant m(z)\left(\nu_{r-}(z) \leqslant m(z)\right)$, и положительным (отрицательным) в некоторой левой окрестности точки $z$, если $\nu_{l+}(z) \leqslant m(z)$ $\left(\nu_{l-}(z) \leqslant m(z)\right)$.

Наконец, выбираем тот набор полиномов $\pm(x-z)^{m}, m=m(z), z=z_{l}$, $l=i, i+1, \ldots, j$, произведение которых с учетом возможных дополнительных множителей вида $\left(x-z_{l} \pm \delta\right)$ дает полином с наименьшей суммарной степенью

$$
\gamma\left(z_{i}\right)+\gamma\left(z_{i+1}\right)+\cdots+\gamma\left(z_{j}\right)
$$

здесь, как и выше, $\gamma(z)$ означает степень полинома $P(x, z)$ оптимального перехода через точку $z$ и равна одному из чисел $m(z)+2, m(z)$ или $m(z)+1$, что зависит от выбранных знаков для всех выражений $\pm(x-z)^{m(z)}, z=z_{l}, l=i, i+1, \ldots, j$; так, пара точек $z_{l}$ и $z_{l+1}$ порождает один дополнительный множитель $\left(x-z_{l}-\delta\right)$, если только соответствующие выражения $\pm(x-z)^{m(z)}$ при $z=z_{l}$ и $z=z_{l+1}$ имеют противоположные знаки на $\left(z_{l}, z_{l+1}\right), l=i, i+1, \ldots, j-1$.

ОПРЕДЕЛЕНИЕ 2. Индексом альтернанса порядка $n, n=0,1, \ldots$, разности $Q(x)-f(x)$ с весом $p=\left(p_{-}, p_{+}\right)$на отрезке $\Delta=[a, b]$ назовем следуюшую величину:

$$
N_{n}(Q, f, p, \Delta)=1+\sum_{i=1}^{k} \gamma_{i}+\sum_{i=0}^{k} m_{i},
$$

где $k$ - мощность множества $F, \gamma_{i}=\gamma\left(z_{i}\right)$ - степень полинома $P\left(x, z_{i}\right)$ оптимального перехода через точку $z_{i} \in F, i=1,2, \ldots, k$, число $m_{i}$ на единицу менњше количества $(+)$ - и $(-)$-интервалов на $\left(z_{i}, z_{i+1}\right), i=0,1, \ldots, k$; при этом считаем $m_{i}=0$, если $\left(z_{i}, z_{i+1}\right)$ пересекается не более чем с одним из множеств $G_{-}$и $G_{+}$; при $n=0$ имеем $k=0$ и индекс альтернанса равен $1+m_{0}$.

\section{§5. Основной результат}

Напомним, что если для данного полинома $Q \in \mathscr{P}_{n}, n=0,1, \ldots$, и непрерывной на отрезке $\Delta$ функции $f(x)$ при заданном на этом отрезке весе $p=\left(p_{-}, p_{+}\right)$ сушествует точка $x \in \Delta_{-}^{\infty}$ с $Q(x)<f(x)$ или точка $x \in \Delta_{+}^{\infty}$ с $Q(x)>f(x)$, то $Q(x)$ является полиномом наилучшего приближения порядка $n$ для $f(x)$ относительно $p$-нормы, т.е. выполняется равенство (1), если только $E_{n}(f, p, \Delta)=\infty$; случай, когда мошность множества $F=\Delta_{-}^{\infty} \cap \Delta_{+}^{\infty}$ всех точек, в любой окрестности каждой из которых не ограничены обе компоненты веса $p$, превосходит порядок полинома наилучшего приближения $n$, также рассмотрен выше.

ТЕОРема 4. Пусть на отрезке $\Delta=[a, b]$ заданы произвольный вес $p=$ $\left(p_{-}, p_{+}\right)$и непрерьвная функция $f(x) ; Q \in \mathscr{P}_{n}, n=0,1, \ldots ;$ мощность множества $F$ не больше $n$, причем $Q(x) \geqslant f(x)$ при $x \in \Delta_{-}^{\infty} u Q(x) \leqslant f(x)$ nрu $x \in \Delta_{+}^{\infty}$.

Тогда для справедливости равенства (1) с данным полиномом $Q(x)$ необходимо и достаточно выполнения хотя бъ одного из следующих условий:

1) множество $E_{-} \cap E_{+}$содержит некоторую точку относительно ее односторонних окрестностей; 
2) найдется точка р-экстремума полной степени для разности $Q(x)-$ $f(x)$ на отрезке $\Delta$

3) индекс альтернанса разности $Q(x)-f(x)$ с весом $p$ на отрезке $\Delta$ удовлетворяет неравенству $N_{n}(Q, f, p, \Delta) \geqslant n+2$.

ДокАЗАТЕЛЬСтво. Необходимость. Если $|Q-f|_{p}=0$, то, очевидно, выполняется условие 1). Пусть $|Q-f|_{p}>0$, вьполняется равенство (1) и не вьполняются условия 1)-3).

Тогда не существует $z \in \Delta$ с $z \in E_{-} \cap E_{+}$относительно ее односторонних окрестностей и на $\Delta$ нет точек $p$-экстремума полной степени разности $Q(x)-f(x)$, а поэтому можно определить индекс альтернанса $N_{n}(Q, f, p, \Delta)$.

Так как не выполняется условие 3$)$, имеем $N_{n}(Q, f, p, \Delta)<n+2$.

Пусть $z_{1}<z_{2}<\cdots<z_{k}$ суть все точки множества $F, z_{0}=a, z_{k+1}=b$; $z_{0} \leqslant z_{1} ; z_{k} \leqslant z_{k+1}$.

На каждом из интервалов $\left(z_{i}, z_{i+1}\right), i=0,1, \ldots, k$, пересекающемся с множеством $G_{-} \cup G_{+}$, возьмем $(+)$- и $(-)$-интервалы

$$
\left(z_{i}, t_{1}^{(i)}\right), \quad\left(t_{1}^{(i)}, t_{2}^{(i)}\right), \quad \ldots, \quad\left(t_{m_{i}}^{(i)}, z_{i+1}\right)
$$

так как не выполняется условие 1$)$, их конечное число (пусть на $\left(z_{i}, z_{i+1}\right)$ получилось $\left(m_{i}+1\right)$ интервалов).

Для каждой точки $z_{i}, i=1,2, \ldots, k$, построим свой полином $P\left(x, z_{i}\right)$ степени $\gamma_{i}$ оптимального перехода через точку $z_{i}$; при этом берем $\delta>0$ (когда $\gamma_{i}=$ $\gamma\left(z_{i}\right) \neq m\left(z_{i}\right)$, необходимо брать дополнительные нули $z_{i}-\delta$ и/или $z_{i}+\delta$ в соответствии с определением этого полинома) такое, что $t_{m_{i-1}}^{(i-1)}<z_{i}-\delta, z_{i}+\delta<t_{1}^{(i)}$ и $\left[z_{i}-\delta, z_{i}\right) \cap G_{ \pm}=\varnothing$ и/или $\left(z_{i}, z_{i}+\delta\right] \cap G_{ \pm}=\varnothing$. В случае сушествования это значение $\delta>0$ фиксируем и обозначим через $\tau$. Если при этом точка $z_{i}+\tau$ принадлежит $(+)$-интервалу, то вместо него получим два новых интервала: ( - -интервал $\left(z_{i}, z_{i}+\tau\right)$ и $(+)$-интервал $\left(z_{i}+\tau, t_{1}^{(i)}\right)$; если же эта точка $z_{i}+\tau$ принадлежит $(-)$-интервалу, то получим $(+)$-интервал $\left(z_{i}, z_{i}+\tau\right)$ и $(-)$-интервал $\left(z_{i}+\tau, t_{1}^{(i)}\right)$; аналогично поступаем с точкой $z_{i}-\tau$. Построим теперь полиномы вида

$$
P_{i}(x)= \pm\left(x-t_{1}^{(i)}\right)\left(x-t_{2}^{(i)}\right) \cdots\left(x-t_{m i}^{(i)}\right), \quad i=0,1, \ldots, k
$$

причем знак + или - выбирается так, что $P_{i}(x)<0$ на $(+)$-интервалах и $P_{i}(x)>0$ на (-)-интервалах из промежутка $\left(z_{i}, z_{i+1}\right)$.

Взяв произведение всех полиномов $P_{i}(x), i=0,1, \ldots, k$, и всех полиномов $P\left(x, z_{i}\right), i=1,2, \ldots, k$, получим некоторый алгебраический полином

$$
R(x)= \pm \prod_{i=1}^{k} P\left(x, z_{i}\right) \prod_{i=0}^{k} P_{i}(x)
$$

степени

$$
\sum_{i=1}^{k} \gamma_{i}+\sum_{i=0}^{k} m_{i}=N_{n}(Q, f, p, \Delta)-1 \leqslant n
$$


Чтобы выбрать знак + или - в равенстве $(15)$ для $R(x)$, заметим, что на отрезке $\Delta$, очевидно, сушествует хотя бы одна точка множества $\Delta\left(p_{-}\right) \cup \Delta\left(p_{+}\right)$. Если она совпадает с одной из точек $z_{i} \in F$, то знак выбираем так, чтобы $R(x)$ и полином $P\left(x, z_{i}\right)$ имели одинаковые знаки в окрестности точки $z_{i}$; в противном случае имеется (+)- или (-)-интервал, который содержит эту точку $p$-экстремума или для которого она служит одним из концов. И тогда знак + или - в (15) выбираем таким образом, чтобы на этом интервале вьполнялось неравенство $R(x)<0$, если он служит $(+)$-интервалом, и неравенство $R(x)>0$, если он окажется $(-)$-интервалом.

Так как по построению знаки каждого полинома $P\left(x, z_{i}\right)$ согласованы со знаками полинома $P_{i-1}(x)$ на промежутке $\left(z_{i-1}, z_{i}\right)$ и со знаками полинома $P_{i}(x)$ на $\left(z_{i}, z_{i+1}\right)$, то $R(x)<0$ на всех $(+)$-интервалах и $R(x)>0$ на всех $(-)$-интервалах отрезка $\Delta$.

ЗАмЕчаниЕ 3. Согласованности знаков полинома $R(x)$ на $(+)-$ и $(-)$-интервалах можно добиться и другим путем. Сначала с помощью простых нулей в точках $t_{j}^{i}, j=1,2, \ldots, m_{i}, i=0,1, \ldots, k$, простых (для нечетных $m\left(z_{i}\right)$ ) или двойных (для четных $m\left(z_{i}\right)$ ) нулей в точках $z_{i}, i=1,2, \ldots, k$, а также простых нулей в возможных дополнительных точках $z_{i}-\tau$ и/или $z_{i}+\tau, i=1,2, \ldots, k$, строим вспомогательный полином $R_{1}(x)$, имеющий требуемые для $R(x)$ знаки на $(+)-$ и $(-)$-интервалах. Затем умножаем $R_{1}(x)$ на неотрицательные множители вида $\left(x-z_{i}\right)^{m\left(z_{i}\right)-1}$ для простых нулей среди $z_{i}$ (что соответствует нечетному $m\left(z_{i}\right)$ ) или вида $\left(x-z_{i}\right)^{m\left(z_{i}\right)-2}$ для двойных нулей среди $z_{i}$ (что соответствует четномy $\left.m\left(z_{i}\right)\right)$.

Чтобы получить противоречие с допушением, остается выбрать достаточно малое число $h>0$ так, чтобы выполнялось неравенство

$$
|(Q+h R)-f|_{p, \Delta}<|Q-f|_{p, \Delta}=|Q-f|_{p}
$$

Для этого отрезок $\Delta$ разобьем на три групшы подотрезков: окрестности точек $z_{i}$, окрестности точек $t_{j}^{i}$ и $z_{i} \pm \tau$ и отрезки, которые остаются между указанными окрестностями. Таких возможных подотрезков конечное число. Покажем, что на каждом таком подотрезке $[\alpha, \beta]$ при достаточно малом $h>0$ выполняется неравенство

$$
\sup \{|(Q+h R-f, p)(x)|: x \in[\alpha, \beta]\}<|Q-f|_{p} .
$$

Рассмотрим сначала окрестность точки $z_{i}, i=1,2, \ldots, k$, для определенности - правую ее окрестность $\left[z_{i}, z_{i}+\delta\right]$ с достаточно малым $\delta>0$.

Если $\gamma_{i}=\gamma\left(z_{i}\right)$ - степень полинома $P\left(x, z_{i}\right)$ оптимального перехода через точку $z_{i}$, то существует значение $m=m\left(z_{i}\right)$ (решение соответствуюшей системы неравенств из определения такого полинома), при котором $\gamma_{i}$ равно одному из значений $m+2, m+1$ или $m$. Тогда $\nu_{r-}\left(z_{i}\right) \leqslant m<\mu_{r+}\left(z_{i}\right)$ или $\nu_{r+}\left(z_{i}\right) \leqslant m<\mu_{r-}\left(z_{i}\right)$ (при этом правая часть неравенств опускается, если соответствующее $\mu_{r+}\left(z_{i}\right)$ или $\mu_{r-}\left(z_{i}\right)$ не существует).

В первом случае $z_{i}$ является левым концом $(+)$-интервала и существуют сколь угодно малые $\alpha>0$ и $\delta>0$, для которых при этом $m=m\left(z_{i}\right)$ выполняется неравенство

$$
\sup \left\{\left(f(x)-Q(x)+\alpha\left|x-z_{i}\right|^{m}\right) p_{-}(x): x \in\left[z_{i}, z_{i}+\delta\right]\right\}<|Q-f|_{p}
$$


Тогда при $x \in\left[z_{i}, z_{i}+\delta\right]$ для $h>0$ c $h|R(x)| \leqslant \alpha\left|x-z_{i}\right|^{m}$ получим

$$
\begin{aligned}
& (Q(x)+h R(x)-f(x))^{-} p_{-}(x)=\max \left\{0,(f(x)-Q(x)-h R(x)) p_{-}(x)\right\} \\
& \quad \leqslant \max \left\{0,\left(f(x)-Q(x)+\alpha\left|x-z_{i}\right|^{m}\right) p_{-}(x)\right\} \\
& \quad \leqslant \max \left\{0, \sup \left\{\left(f(x)-Q(x)+\alpha\left|x-z_{i}\right|^{m}\right) p_{-}(x): x \in\left[z_{i}, z_{i}+\delta\right]\right\}\right\} \\
& \quad<|Q-f|_{p} .
\end{aligned}
$$

Далее, если точка $z_{i} \notin \Delta\left(p_{+}\right)$относительно ее правой окрестности, то при $x \in\left[z_{i}, z_{i}+\delta\right]$ с достаточно малым $\delta>0$ полином $R(x) \leqslant 0$ (так как $z_{i}$ служит левым концом (+)-интервала), а поэтому

$(Q(x)+h R(x)-f(x)) p_{+}(x) \leqslant \sup \left\{(Q(x)-f(x)) p_{+}(x): x \in\left[z_{i}, z_{i}+\delta\right]\right\}<|Q-f|_{p}$.

Пусть теперь $z_{i} \in \Delta\left(p_{+}\right)$. Тогда по определению величины $\mu_{r+}\left(z_{i}\right)$ при $m=m\left(z_{i}\right)$ для любой последовательности точек $y_{j} \rightarrow z_{i}, j \rightarrow \infty, y_{j} \geqslant z_{i}$, такой, что

$$
\left(Q\left(y_{j}\right)-f\left(y_{j}\right)\right) p_{+}\left(y_{j}\right) \rightarrow|Q-f|_{p},
$$

последовательность $\left(y_{j}-z_{i}\right)^{m} p_{+}\left(y_{j}\right)$ не стремится к нулю при $j \rightarrow \infty$. Поэтому найдется такое $\delta>0$, что

$$
\sup \left\{(Q(x)+h R(x)-f(x)) p_{+}(x): x \in\left[z_{i}, z_{i}+\delta\right]\right\}<|Q-f|_{p} .
$$

Действительно, в противном случае из $R(x)<0$ при $x \in\left(z_{i}, z_{i}+\delta\right]$ для всех достаточно малых $\delta>0$ получим

$$
\sup \left\{(Q(x)+h R(x)-f(x)) p_{+}(x): x \in\left[z_{i}, z_{i}+\delta\right]\right\}=|Q-f|_{p} .
$$

Тогда найдется последовательность точек $y_{j} \rightarrow z_{i}, j \rightarrow \infty, y_{j} \geqslant z_{i}$, такая, что

$$
\left(Q\left(y_{j}\right)+h R\left(y_{j}\right)-f\left(y_{j}\right)\right) p_{+}\left(y_{j}\right) \rightarrow|Q-f|_{p}, \quad j \rightarrow \infty,
$$

при этом

$$
\left(Q\left(y_{j}\right)+h R\left(y_{j}\right)-f\left(y_{j}\right)\right) p_{+}\left(y_{j}\right) \leqslant\left(Q\left(y_{j}\right)-f\left(y_{j}\right)\right) p_{+}\left(y_{j}\right) \leqslant|Q-f|_{p} .
$$

Следовательно, для этой же последовательности $y_{j}$ имеем

$$
\left(Q\left(y_{j}\right)-f\left(y_{j}\right)\right) p_{+}\left(y_{j}\right) \rightarrow|Q-f|_{p}, \quad j \rightarrow \infty,
$$

а поэтому (взяв разность) при $j \rightarrow \infty$ получим

$$
h R\left(y_{j}\right) p_{+}\left(y_{j}\right) \rightarrow 0 .
$$

Так как $R(x)$ и $\left(x-z_{i}\right)^{m}, m=m\left(z_{i}\right)$, - бесконечно малые функции одного порядка при $x \rightarrow z_{i}$, то также имеем

$$
\left(y_{j}-z_{i}\right)^{m} p_{+}\left(y_{j}\right) \rightarrow 0, \quad j \rightarrow \infty,
$$

что противоречит неравенству $m=m\left(z_{i}\right)<\mu_{r+}\left(z_{i}\right)$. 
Из (18) и (19) получим (17) для $[\alpha, \beta]=\left[z_{i}, z_{i}+\delta\right]$ с достаточно малым $\delta>0$ в случае $\nu_{r-}\left(z_{i}\right) \leqslant m\left(z_{i}\right)<\mu_{r+}\left(z_{i}\right) ;$ другой возможный случай $\nu_{r+}\left(z_{i}\right) \leqslant m\left(z_{i}\right)<$ $\mu_{r-}\left(z_{i}\right)$ рассматривается вполне аналогично.

Случаи окрестностей точек вида $t_{j}^{(i)}$ и точек вида $z_{i} \pm \tau$ рассматриваются аналогично друг другу; пусть $t$ - точка одного из этих двух видов. Так как $t \notin G_{-} \cup G_{+}$, найдется достаточно малое $\delta>0$ такое, что

$$
\sup \{|(Q-f, p)(x)|: x \in[t-\delta, t+\delta]\}<|Q-f|_{p}
$$

Пусть, например, $t$ служит левым концом $(+)$-интервала и правым концом $(-)$-интервала. Тогда $R(x) \leqslant 0$ при $x \in[t, t+\delta]$, поэтому из (20) получим

$$
\begin{aligned}
& \sup \left\{(Q(x)+h R(x)-f(x)) p_{+}(x): x \in[t, t+\delta]\right\} \\
& \quad \leqslant \sup \left\{(Q(x)-f(x)) p_{+}(x): x \in[t, t+\delta]\right\}<|Q-f|_{p} .
\end{aligned}
$$

Если $t \notin \Delta_{-}^{\infty}$, то возьмем достаточно малое $\delta>0$, для которого $p_{-}(x)$ ограничена при $x \in[t, t+\delta]$. Тогда с использованием (20) имеем

$$
\begin{aligned}
\sup \left\{(f(x)-Q(x)-h R(x)) p_{-}(x)\right. & : x \in[t, t+\delta]\} \\
\leqslant & \sup \left\{(Q(x)-f(x)) p_{-}(x): x \in[t, t+\delta]\right\} \\
+h \sup \left\{|R(x)| p_{-}(x): x\right. & \in[t, t+\delta]\}<|Q-f|_{p}
\end{aligned}
$$

при достаточно малом $h>0$.

Если $t \in \Delta_{-}^{\infty}$, то неравенство $Q(t)<f(t)$ исключено по условию; равенство $Q(t)=f(t)$ означало бы $t \in G_{-}$, чего не может быть для рассматриваемого случая точки $t$; остается $Q(t)>f(t)$. Поэтому в силу непрерьвности $Q(x)>f(x)$ при $x \in[t, t+\delta]$ с достаточно малым $\delta>0$ (которое выбирается так, чтобы вьполнялось и неравенство (20)).

Выберем теперь число $h>0$ столь мальм, чтобы при $x \in[t, t+\delta]$ выполнялось неравенство

$$
h|R(x)|<\min \{Q(x)-f(x): x \in[t, t+\delta]\} .
$$

Тогда при всех $x \in[t, t+\delta]$ имеем

$$
(Q(x)+h R(x)-f(x))^{-} p_{-}(x)=0 .
$$

Итак, сушествуют достаточно малые $\delta>0$ и $h>0$ такие, что

$$
\sup \{|(Q+h R-f, p)(x)|: x \in[t, t+\delta]\}<|Q-f|_{p} .
$$

Аналогичная же оценка получается в случае левой окрестности $[t-\delta, t]$ точки $t$. Наконец, рассмотрим отрезки $[\alpha, \beta]$, расположенные на данном (+)- или (-)-интервале, концы $\alpha$ и $\beta$ которых являются точками вида $t_{j}^{(i)} \pm \delta$ или $\left(z_{i} \pm \tau\right) \pm \delta$; пусть для определенности $[\alpha, \beta]$ расположен на одном из $(+)$-интервалов, тогда $R(x)<0$ при $x \in[\alpha, \beta]$. 
Если $[\alpha, \beta] \cap \Delta\left(p_{+}\right)=\varnothing$, то, очевидно,

$$
\sup \left\{(Q(x)+h R(x)-f(x)) p_{+}(x): x \in[\alpha, \beta]\right\}<|Q-f|_{p} .
$$

Пусть $[\alpha, \beta] \cap \Delta\left(p_{+}\right) \neq \varnothing$. Так как $R(x)<0$ при $x \in[\alpha, \beta]$, левая часть неравенства (21) не может быть строго больше его правой части. Поэтому допустим, что в этом случае вместо неравенства в (21) выполняется равенство. При этом не сушествует точки $x_{0} \in[\alpha, \beta]$ такой, что

$$
\left(Q\left(x_{0}\right)+h R\left(x_{0}\right)-f\left(x_{0}\right)\right) p_{+}\left(x_{0}\right)=|Q-f|_{p},
$$

так как в случае существования такой точки получили бы $p_{+}\left(x_{0}\right) \neq 0$ (у нас $\left.|Q-f|_{p}>0\right)$, что ввиду $R\left(x_{0}\right)<0$ приводит к противоречию

$$
\left(Q\left(x_{0}\right)+h R\left(x_{0}\right)-f\left(x_{0}\right)\right) p_{+}\left(x_{0}\right)<\left(Q\left(x_{0}\right)-f\left(x_{0}\right)\right) p_{+}\left(x_{0}\right) \leqslant|Q-f|_{p} .
$$

Следовательно, при $x_{0} \in \Delta\left(p_{+}\right) \cap[\alpha, \beta]$ найдется последовательность точек $y_{j} \rightarrow x_{0}, j \rightarrow \infty, y_{j} \neq x_{0}$, такая, что

$$
\left(Q\left(y_{j}\right)+h R\left(y_{j}\right)-f\left(y_{j}\right)\right) p_{+}\left(y_{j}\right) \rightarrow|Q-f|_{p}
$$

при $j \rightarrow \infty$.

Тогда из неравенств

$$
\left(Q\left(y_{j}\right)+h R\left(y_{j}\right)-f\left(y_{j}\right)\right) p_{+}\left(y_{j}\right) \leqslant\left(Q\left(y_{j}\right)-f\left(y_{j}\right)\right) p_{+}\left(y_{j}\right) \leqslant|Q-f|_{p}
$$

вытекает, что при $j \rightarrow \infty$

$$
\left(Q\left(y_{j}\right)-f\left(y_{j}\right)\right) p_{+}\left(y_{j}\right) \rightarrow|Q-f|_{p}
$$

Из $(22),(23)$ и $R\left(x_{0}\right) \neq 0$ следует $p_{+}\left(y_{j}\right) \rightarrow 0$ при $j \rightarrow 0$, что противоречит (23), так как $|Q-f|_{p}>0$ и $Q(x)-f(x)$ непрерывна.

Итак, если отрезок $[\alpha, \beta]$ расположен на $(+)$-интервале, то выполняется строгое неравенство (21).

Рассмотрим теперь случай отрезка $[\alpha, \beta]$, расположенного на $(+)$-интервале, для другой компоненты $p_{-}(x)$ веса.

Если $[\alpha, \beta] \cap \Delta_{-}^{\infty}=\varnothing$, то (так как $\left.[\alpha, \beta] \cap \Delta\left(p_{-}\right)=\varnothing\right)$ имеем

$$
\begin{aligned}
\sup \{( & \left.f(x)-Q(x)-h R(x)) p_{-}(x): x \in[\alpha, \beta]\right\} \\
& \leqslant \sup \left\{(f(x)-Q(x)) p_{-}(x): x \in[\alpha, \beta]\right\}+h \sup \left\{|R(x)| p_{-}(x): x \in[\alpha, \beta]\right\} \\
& <|Q-f|_{p}
\end{aligned}
$$

при достаточно малом $h>0$.

Поэтому пусть $[\alpha, \beta] \cap \Delta_{-}^{\infty} \neq \varnothing$. Тогда множество $D=\left\{x \in[\alpha, \beta] \cap \Delta_{-}^{\infty}\right.$ : $Q(x)>f(x)\}$ замкнуто. 
Действительно, $\Delta_{-}^{\infty}$ замкнуто и по условию при $x \in \Delta_{-}^{\infty}$ выполняется неравенство $Q(x) \geqslant f(x)$, причем отрезок $[\alpha, \beta]$ расположен на $(+)$-интервале, а поэтому не пересекается с $\Delta_{-}^{\infty}(Q=f) \subset G_{-}$.

Следовательно, предельные точки множества $D$ (в случае их существования) принадлежат самому $D$.

Тогда в силу непрерывности сушествует $\min \{(Q(x)-f(x)): x \in D\}=\eta>0$.

Пусть теперь $J$ - объединение конечного числа таких интервалов, что $D \subset J$ и при $x \in J \cap[\alpha, \beta]$ выполняется неравенство $Q(x)-f(x)>(1 / 2) \eta$.

Возьмем $h>0$ столь мальм, чтобы вьполнялось неравенство $h|R(x)|<(1 / 2) \eta$ при $x \in J \cap[\alpha, \beta]$, тогда при всех таких $x$ имеем

$$
(Q(x)+h R(x)-f(x))^{-} p_{-}(x)=0 .
$$

На оставшемся множестве $I=[\alpha, \beta] \backslash J$ компонента $p_{-}(x)$ веса ограничена и $[\alpha, \beta] \cap \Delta\left(p_{-}\right)=\varnothing$, а поэтому

$$
\begin{aligned}
\sup \{( & \left.f(x)-Q(x)-h R(x)) p_{-}(x): x \in I\right\} \\
& \leqslant \sup \left\{(f(x)-Q(x)) p_{-}(x): x \in I\right\}+h \sup \left\{|R(x)| p_{-}(x): x \in I\right\} \\
& <|Q-f|_{p}
\end{aligned}
$$

при достаточно малом $h>0$ (с учетом предыдущих значений $h$ ).

Отсюда и из (21) вытекает неравенство (17) в случае отрезка $[\alpha, \beta]$, расположенного на некотором (+)-интервале.

Случай, когда отрезок $[\alpha, \beta]$ расположен на одном из (-)-интервалов, рассматривается вполне аналогично.

Итак, если выполняется равенство (1) и не выполняются условия 1) и 2), то вьполняется условие 3 ), т.е. необходимость доказана.

Достаточность. Если $|Q-f|_{p}=0$, то равенство (1), очевидно, выполняется. Поэтому пусть $|Q-f|_{p}>0$.

Достаточность каж дого из условий 1) и 2) для выполнения равенства (1) доказана выше (теоремы 2 и 3 соответственно).

Остается доказать достаточность условия 3) для выполнения (1), причем достаточно считать, что выполняется условие 3 ) и не выполняются условия 1) и 2) (так как достаточность последних уже доказана). Тогда можно определить индекс альтернанса. Пусть индекс альтернанса порядка $n$ разности $Q(x)-f(x)$ с весом $p=\left(p_{-}, p_{+}\right)$на отрезке $\Delta=[a, b]$ имеет следуюший вид:

$$
N_{n}(Q, f, p, \Delta)=1+\sum_{i=1}^{k} \gamma_{i}+\sum_{i=0}^{k} m_{i},
$$

т.е. множество $F=\Delta_{-}^{\infty} \cap \Delta_{+}^{\infty}$ состоит из $k$ точек

$$
z_{1}<z_{2}<\cdots<z_{k}, \quad z_{1} \geqslant a=z_{0}, \quad z_{k} \leqslant b=z_{k+1},
$$

для которых на каждом из промежутков

$$
\left[z_{0}, z_{1}\right), \quad\left(z_{1}, z_{2}\right), \ldots, \quad\left(z_{k}, z_{k+1}\right]
$$


найдутся $m_{i}, i=0,1, \ldots, k$, точек $t_{1}^{(i)}<t_{2}^{(i)}<\cdots<t_{m_{i}}^{(i)}$ таких, что $t_{j}^{(i)} \notin G_{-} \cup G_{+}$, $j=1,2, \ldots, m_{i}$, а интервалы

$$
\left(z_{i}, t_{1}^{(i)}\right), \quad\left(t_{1}^{(i)}, t_{2}^{(i)}\right), \quad \ldots, \quad\left(t_{m_{i}}^{(i)}, z_{i+1}\right)
$$

с чередованием пересекаются то с множеством $G_{-}$, то с множеством $G_{+}$; далее, $\gamma_{i}=\gamma\left(z_{i}\right)$ равно степени полинома $P\left(x, z_{i}\right)$ оптимального перехода через точку $z_{i}$, $i=1,2, \ldots, k$, причем $\gamma\left(z_{i}\right)$ равно одному из чисел $m\left(z_{i}\right)+2, m\left(z_{i}\right)+1$ и $m\left(z_{i}\right)$ в зависимости от вида $P\left(x, z_{i}\right)$.

Так как не выполняется условие 2), при $n=0$ имеем $k=0$ и $N_{0}(Q, f, p, \Delta)=$ $1+m_{0}$.

Допустим, что существует такой полином $P \in \mathscr{P}_{n}$, что выполняется неравенство (6). Тогда, так как еще $z_{i} \in F$, при $m=m\left(z_{i}\right)$, как и выше, получим

$$
P(x)=Q(x)+a_{m}\left(x-z_{i}\right)^{m}+a_{m+1}\left(x-z_{i}\right)^{m+1}+\cdots+a_{n}\left(x-z_{i}\right)^{n} .
$$

Пусть сначала на $\left(z_{i}, z_{i+1}\right)$ имеются $(-)$ - и/или $(+)$-интервалы, для определенности $\left(z_{i}, t_{1}^{(i)}\right)$ является $(-)$-интервалом и точка $z_{i} \in E_{+}$относительно ее правых окрестностей. Тогда согласно определению полинома $P\left(x, z_{i}\right)$ оптимального перехода через точку $z_{i}$ берется дополнительная точка $z_{i}+\delta$ со сколь угодно малым $\delta>0$.

Так как при этом $\left(z_{i}, t_{1}^{(i)}\right)$ является $(-)$-интервалом, найдется точка $y \in$ $\left(z_{i}+\delta, t_{1}^{(i)}\right) \cap G_{-}$, в которой $Q(y) \leqslant P(y)$.

Действительно, из $y \in G_{-}$имеем $f(y) \geqslant Q(y)$. Поэтому при $y \in \Delta_{-}^{\infty}$, если допустить $Q(y)>P(y)$, найдется последовательность $y_{j} \rightarrow y, j \rightarrow \infty$, с $p_{-}\left(y_{j}\right) \rightarrow+\infty$ и

$$
\left(f\left(y_{j}\right)-P\left(y_{j}\right)\right) p_{-}\left(y_{j}\right) \geqslant\left(Q\left(y_{j}\right)-P\left(y_{j}\right)\right) p_{-}\left(y_{j}\right) \rightarrow+\infty
$$

при $j \rightarrow \infty$, что противоречит конечности $|P-f|_{p}$ (ввиду (6)). Если же $y \notin \Delta_{-}^{\infty}$, то $y \in \Delta\left(p_{-}\right)$, а поэтому из (6) следует требуемое $Q(y)<P(y)$.

С другой стороны, так как $z_{i} \in E_{+}$относительно ее правых окрестностей и вьполняется (6), найдется такая точка $y_{1} \in\left(z_{i}, z_{i}+\delta\right)$, что $Q\left(y_{1}\right)>P\left(y_{1}\right)$.

Следовательно, в рассматриваемом случае на $\left(z_{i}, t_{1}^{(i)}\right)$ найдется еще одна точка $x$, в которой $Q(x)=P(x)$.

Если точка $z_{i} \notin E_{+}$относительно ее правых окрестностей, то подобной точки $x \in\left(z_{i}, t_{1}^{(i)}\right)$ может не существовать, что соответствует определению степени $\gamma\left(z_{i}\right)$ полинома $P\left(x, z_{i}\right)$.

Аналогично обстоит дело в левой окрестности точки $z_{i}$.

Итак, каждой точке $z_{i}, i=1,2, \ldots, k$, которая является конщом $(-)$ - или $(+)$-интервалов, соответствует $\gamma_{i}=\gamma\left(z_{i}\right)$ общих точек полиномов $Q(x)$ и $P(x)$.

Кроме того, на $\left(z_{i}, z_{i+1}\right), i=0,1, \ldots, k$, полиномы $Q(x)$ и $P(x)$ имеют еще не менее $m_{i}$ общих точек, так как при $x \in G_{-}$выполняется неравенство $Q(x) \leqslant P(x)$, а при $x \in G_{+}-$неравенство $Q(x) \geqslant P(x)$. 
Пусть теперь $1 \leqslant i<j \leqslant k$ и на $\left(z_{i}, z_{j}\right)$ нет $(-)$ - и $(+)$-интервалов (при этом возможны равенства $z_{i}=z_{0}=a$ или $\left.z_{j}=z_{k+1}=b\right)$, причем $z_{i}$ и жат $\Delta\left(p_{-}\right) \cup \Delta\left(p_{+}\right)$или являются концами $(-)$- и/или $(+)$-интервалов (очевидно, имеется хотя бы одна точка множества $\left.\Delta\left(p_{-}\right) \cup \Delta\left(p_{+}\right)\right)$.

Так как все точки $z=z_{i}, z_{i+1}, \ldots, z_{j}$ принадлежат множеству $F$ и выполняется неравенство (6), для каждой точки $z$ из них и соответствующей степени $m=m(z)$ имеем

$$
P(x)=Q(x)+a_{m}(x-z)^{m}+\cdots+a_{n}(x-z)^{n}, \quad m \geqslant 1 .
$$

Тогда при достаточно малом $\delta>0$ для всех $x \in(z, z+\delta)$ либо $P(x)<Q(x)$ и тогда $a_{m}(x-z)^{m}<0$, либо $P(x)>Q(x)$ и тогда $a_{m}(x-z)^{m}>0$.

Если $a_{m}(x-z)^{m}<0$ при $x \in(z, z+\delta)$, то по определению величины $\nu_{r-}(z)$ при $m<\nu_{r-}(z)$, как и вьшше (см. (13)), придем к противоречию с неравенством (6).

Если же $a_{m}(x-z)^{m}>0$ при $x \in(z, z+\delta)$, то аналогично при $m<\nu_{r+}(z)$ придем к такому же противоречию.

Аналогично обстоит дело в левой окрестности точки $z$.

Следовательно, при выполнении неравенства (6) для каждой из точек $z=z_{i}$, $z_{i+1}, \ldots, z_{j}$ имеем

$$
P(x)-Q(x)=O\left((x-z)^{m(z)}\right), \quad x \rightarrow z,
$$

и выполняется одно из следующих четырех условий (при этом для каждого условия в первой строке считаем $x \in(z, z+\delta)$, а во второй строке $-x \in(z-\delta, z)$ при достаточно малом $\delta>0)$ :

$$
\begin{array}{r} 
\begin{cases}\nu_{r-}(z) \leqslant m(z), & P(x)-Q(x)<0, \\
\nu_{l-}(z) \leqslant m(z), & P(x)-Q(x)<0\end{cases} \\
\begin{cases}\nu_{r-}(z) \leqslant m(z), & P(x)-Q(x)<0, \\
\nu_{l+}(z) \leqslant m(z), & P(x)-Q(x)>0 ;\end{cases} \\
\begin{cases}\nu_{r+}(z) \leqslant m(z), & P(x)-Q(x)>0, \\
\nu_{l-}(z) \leqslant m(z), & P(x)-Q(x)<0\end{cases} \\
\begin{cases}\nu_{r+}(z) \leqslant m(z), & P(x)-Q(x)>0, \\
\nu_{l+}(z) \leqslant m(z), & P(x)-Q(x)>0\end{cases}
\end{array}
$$

Следовательно, полином $P(x)-Q(x)$ в окрестности каждой из этих точек $z=z_{l}$, $l=i, i+1, \ldots, j$, имеет такие же знаки, что и соответствуюший полином $P(x, z)$ оптимального перехода через точку $z$, и, более того, наименьшие целые решения систем неравенств, которым удовлетворяют числа $m(z)$, образуют степени $\gamma(z)$ этих полиномов $P(x, z)$. Отсюда число нулей (с учетом их кратности) полинома $P(x)-Q(x)$ на промежутке $\left[z_{i}, z_{j}\right]$ не меньше числа нулей произведения

$$
\prod_{l=i}^{j} P\left(x, z_{l}\right)
$$

на этом промежутке. 
Если при этом концы промежутка $z_{i}$ и $z_{j}$ таковы, что $P\left(x, z_{i}\right)$ содержит сомножитель вида $\left(x-z_{i}+\delta\right)$ и/или $P\left(x, z_{j}\right)$ содержит сомножитель вида $\left(x-z_{j}-\delta\right)$, то, как показано вьше, им соответствуют дополнительные нули полинома $P(x)-Q(x)$ на $\left(t_{m_{i-1}}^{(i-1)}, z_{i}\right)$ и/или на $\left(z_{j}, t_{1}^{(j)}\right)$; поэтому число нулей полинома $P(x)-Q(x)$ на $\left[z_{i}, z_{j}\right]$ вместе с дополнительными нулями не менее

$$
\gamma\left(z_{i}\right)+\gamma\left(z_{i+1}\right)+\cdots+\gamma\left(z_{j}\right)
$$

Заметим, что в этом случае

$$
m_{i}=m_{i+1}=\cdots=m_{j-1}=0 .
$$

Следовательно, с учетом предыдущих случаев полином $P(x)-Q(x)$ степени не вьше $n$ имеет по меньшей мере

$$
\sum_{i=1}^{k} \gamma_{i}+\sum_{i=0}^{k} m_{i}=N_{n}(Q, f, p, \Delta)-1
$$

нулей, чего не может быть, так как $N_{n}(Q, f, p, \Delta)-1 \geqslant n+1$ и вьполняется неравенство (6).

Теорема 4 доказана.

\section{§6. Некоторые следствия}

Ниже будем считать, что исключается тривиальный случай, когда заданные непрерывная на отрезке $\Delta=[a, b]$ функция $f(x)$ и полином $Q \in \mathscr{P}_{n}$ тождественно совпадают на $\Delta$.

В качестве следствия теоремы 4 получим характеристическое свойство полинома наилучшего приближения непрерывной функции в равномерной метрике с интерполяцией и выясним для этого случая значения параметров, входящих в теоремy 4 .

Для заданных на отрезке $\Delta$ различных точек $z_{1}<z_{2}<\cdots<z_{k}, k \geqslant 1$, положим $p_{-}\left(z_{i}\right)=p_{+}\left(z_{i}\right)=+\infty, p_{-}(x)=p_{+}(x)=1$ при $x \neq z_{i}, x \in \Delta, i=1,2, \ldots, k$, и вес $p=\left(p_{-}, p_{+}\right)$с такими компонентами ниже назовем интерполирующим весом.

Тогда условие 3$)$ теоремы 4 с интерполирующим весом $p=\left(p_{-}, p_{+}\right)$служит характеристическим свойством полинома $Q \in \mathscr{P}_{n}, k \leqslant n$, наилучшего равномерного приближения непрерывной на $\Delta$ функции $f(x)$ в смысле

$$
\|Q-f\|_{\Delta}=A:=\inf \left\{\|P-f\|_{\Delta}: P \in \mathscr{P}_{n}, P\left(z_{i}\right)=f\left(z_{i}\right), i=1,2, \ldots, k\right\},
$$

$Q\left(z_{i}\right)=f\left(z_{i}\right)$ при $i=1,2, \ldots, k$.

Действительно, пусть для полинома $Q \in \mathscr{P}_{n}$ в случае интерполируюшего веса $p=\left(p_{-}, p_{+}\right)$выполнено условие 3$)$ теоремы 4 . Тогда по теореме 4 вьполняется равенство

$$
|Q-f|_{p, \Delta}=A_{1}:=\inf \left\{|P-f|_{p, \Delta}: P \in \mathscr{P}_{n}\right\}
$$


Докажем, что для этого полинома $Q(x)$ выполняется и условие (24). Так как в случае интерполирующего веса $p=\left(p_{-}, p_{+}\right)$для полиномов $P \in \mathscr{P}_{n}$ с $P\left(z_{i}\right) \neq f\left(z_{i}\right)$ хотя бы в одной точке $z_{i}, i=1,2, \ldots, k$, величина $|P-f|_{p, \Delta}=\infty$, то выполняется равенство

$$
A_{1}=A_{2}:=\inf \left\{|P-f|_{p, \Delta}: P \in \mathscr{P}_{n}, P\left(z_{i}\right)=f\left(z_{i}\right), i=1,2, \ldots, k\right\},
$$

причем $k \leqslant n$. Поэтому $|Q-f|_{p, \Delta}=A_{1}=A_{2}<\infty$, а следовательно, $Q\left(z_{i}\right)=f\left(z_{i}\right)$ при $i=1,2, \ldots, k$.

Ясно, что для интерполируюшего веса $p=\left(p_{-}, p_{+}\right)$и любого полинома $P \in \mathscr{P}_{n}$ с $P\left(z_{i}\right)=f\left(z_{i}\right), i=1,2, \ldots, k$, при $x \in \Delta$ имеем

$$
|P(x)-f(x)|=(P(x)-f(x))^{+}+(P(x)-f(x))^{-}=|(P-f, p)(x)| .
$$

Поэтому $A=A_{2}$. Тогда $\|Q-f\|_{\Delta}=|Q-f|_{p, \Delta}=A_{1}=A_{2}=A$, т.е. для полинома $Q(x)$ выполнено также равенство (24).

Обратно, пусть для данного полинома $Q \in \mathscr{P}_{n}$ выполнено условие (24) (вместе с $Q\left(z_{i}\right)=f\left(z_{i}\right)$ при $\left.i=1,2, \ldots, k\right)$. Докажем, что тогда для интерполируюшего веса $p=\left(p_{-}, p_{+}\right)$выполняется условие 3$)$ теоремы 4 (параллельно выясним в этом случае значения участвующих в теореме 4 параметров).

Как и выше, имеем $|Q-f|_{p, \Delta}=\|Q-f\|_{\Delta}=A=A_{2}=A_{1}$.

Следовательно, по теореме 4 выполняется хотя бы одно из условий 1$)-3)$.

Остается доказать, что условия 1) и 2) теоремы 4 не выполняются (напомним, что считаем $Q(x) \neq f(x)$ хотя бы в одной точке $x \in \Delta)$.

Покажем, что в рассматриваемом случае множество $E_{-} \cap E_{+}=\varnothing$.

Так как

$$
\Delta_{-}^{\infty}=\Delta_{+}^{\infty}=\left\{z_{1}, z_{2}, \ldots, z_{k}\right\}
$$

множества $\Delta_{-}^{\infty}(Q=f)$ и $\Delta_{+}^{\infty}(Q=f)$, очевидно, пусты.

Множества $\Delta\left(p_{-}, n\right)$ и $\Delta\left(p_{+}, n\right)$ также пусты, так как в силу непрерывности $f(x)-Q(x)$ для любой точки $y \in \Delta$ с $y \notin \Delta\left(p_{-}\right) \cup \Delta\left(p_{+}\right)$найдется ее окрестность, во всех точках $x$ которой выполняется строгое неравенство $|f(x)-Q(x)|<$ $|Q-f|_{p, \Delta}$, а поэтому при достаточно малых $\delta>0$ и $\alpha>0$ условия (4) и (5) с $p_{-}(x)=p_{+}(x)=1$ не могут вьполняться (так как $\left.|Q-f|_{p, \Delta}>0\right)$, в противном случае по непрерьвности функции $|f(x)-Q(x)|$ точка $y \in \Delta\left(p_{-}\right) \cup \Delta\left(p_{+}\right)$.

Следовательно, имеем $E_{-}=\Delta\left(p_{-}\right), E_{+}=\Delta\left(p_{+}\right)$.

Так как $f(x) \neq Q(x)$ хотя бы в одной точке $x \in \Delta$ и $f(x)-Q(x)$ непрерьвна на $\Delta$, то, очевидно, множества $\Delta\left(p_{-}\right)$и $\Delta\left(p_{+}\right)$, а следовательно, и множества $E_{-}$и $E_{+}$ не пересекаются, т.е. условие 1$)$ теоремы 4 не выполняется.

Покажем, что условие 2) теоремы 4 также не может выполняться.

Действительно, точка $p$-экстремума полной степени для разности $Q(x)-f(x)$ на $\Delta$ должна принадлежать как множеству $F=\Delta_{-}^{\infty} \cap \Delta_{+}^{\infty}$, так и множеству $\Delta\left(p_{-}\right) \cup \Delta\left(p_{+}\right)$. В нашем случае $F=\left\{z_{1}, z_{2}, \ldots, z_{k}\right\}$, в то время как $z_{i} \notin \Delta\left(p_{-}\right) \cup$ $\Delta\left(p_{+}\right), i=1,2, \ldots, k$.

Действительно, при каждом $i=1,2, \ldots, k$ имеем $Q\left(z_{i}\right)=f\left(z_{i}\right)$, поэтому в силу непрерывности $Q(x)-f(x)$ найдется окрестность точки $z_{i}$, в которой

$$
|Q(x)-f(x)|<\frac{1}{2}|Q-f|_{p, \Delta},
$$


а следовательно, в этой окрестности (для интерполирующего веса) выполняется также неравенство

$$
|(Q-f, p)(x)|<\frac{1}{2}|Q-f|_{p, \Delta} .
$$

Так как полином наилучшего приближения $Q(x)$ для $f(x)$ в $p$-норме не удовлетворяет условиям 1) и 2) теоремы 4 , то согласно этой теореме выполняется условие 3).

Для рассматриваемого интерполирующего веса $p=\left(p_{-}, p_{+}\right)$выясним также параметры, входящие в индекс альтернанса $N_{n}(Q, f, p, \Delta)$ (см. определение 2).

Число $m_{i}$ на единицу меньше количества $(+)$ - и (-)-интервалов на $\left(z_{i}, z_{i+1}\right)$, $i=0,1, \ldots, k, z_{0}=a, z_{k+1}=b$, которые определяются через пересечения с множествами $G_{-}=E_{-}=\Delta\left(p_{-}\right)$и $G_{+}=E_{+}=\Delta\left(p_{+}\right)$; при этом считаем $m_{i}=0$, если $\left(z_{i}, z_{i+1}\right)$ пересекается не более чем с одним из множеств $G_{-}$и $G_{+}, i=1,2, \ldots, k$.

Выясним теперь значения $\gamma_{i}, i=1,2, \ldots, k$.

Точки $z=z_{i} \notin E_{-} \cup E_{+}, i=1,2, \ldots, k$, поэтому величины $\nu_{r \pm}(z)$ и $\nu_{l \pm}(z)$ существуют и, как следует из (7) и (8), в случае интерполирующего веса все они равны 1 . Так как $z=z_{i} \notin \Delta\left(p_{-}\right) \cup \Delta\left(p_{+}\right)$, величины $\mu_{r \pm}(z)$ и $\mu l \pm(z)$ не определяются.

Следовательно, при $z=z_{i}$ для построения полинома оптимального перехода $P(x, z)$ находим наименьшие целые решения $m=m(z)$ четырех систем, образуемых всеми парами разных неравенств из $\nu r \pm(z) \leqslant m, \nu l \pm(z) \leqslant m$. Так как все левые части этих неравенств равны 1 , искомое значение $m(z)$ равно 1 или 2 в зависимости от того, является точка $z$ общим концом разноименных или одноименных среди построенных на $\Delta(+)$ - и $(-)$-интервалов. Отсюда вытекает, что $\gamma_{i}=\gamma\left(z_{i}\right)=m\left(z_{i}\right), i=1,2, \ldots, k$, соответственно равно 1 , если $z_{i}$ - общий конец разноименных, и равно 2 , если $z_{i}$ - общий конец одноименных $(+)-$ и (-)-интервалов.

Рассмотрим теперь другой частньй случай веса $p=\left(p_{-}, p_{+}\right)$с $p_{-}(x)=p_{+}(x)=1$ при всех $x \in \Delta$. В этом случае $p$-норма по данному отрезку, очевидно, совпадает с обычной равномерной нормой по этому отрезку. Множества $\Delta_{-}^{\infty}$ и $\Delta_{+}^{\infty}$ пусты, условия 1) и 2) теоремы 4 отсутствуют, а условие 3 ) принимает вид

$$
N_{n}(Q, f, p, \Delta)=1+m_{0} \geqslant n+2
$$

и означает наличие на отрезке $\Delta$ чебышёвского альтернанса разности $Q(x)-f(x)$ (так как в этом случае, очевидно, $\left.G_{-}=\Delta\left(p_{-}\right), G_{+}=\Delta\left(p_{+}\right)\right)$.

Третье следствие касается односторонних приближений данной непрерывной на отрезке $\Delta$ функции $f(x)$, причем для удобства формулировок тривиальный случай тождественного совпадения данных функции $f(x)$ и полинома $Q(x)$ на всем $\Delta$ снова исключается.

Пусть $p_{-}(x)=+\infty$ и $p_{+}(x)=1$ при всех $x \in \Delta$. Тогда для веса $p=(+\infty, 1)$ с такими компонентами условие 3 ) теоремы 4 преврашается в характеристическое свойство полинома $Q \in \mathscr{P}_{n}$ наилучшего одностороннего (сверху) приближения непрерывной на отрезке $\Delta$ функции $f(x)$ в смысле

$$
\|Q-f\|_{\Delta}=B:=\inf \left\{\|P-f\|_{\Delta}: P \in \mathscr{P}_{n}, P(x) \geqslant f(x), x \in \Delta\right\}
$$

с $Q(x) \geqslant f(x)$ при $x \in \Delta$. 
Действительно, пусть для некоторого полинома $Q \in \mathscr{P}_{n}$ выполнено условие 3 ) теоремы 4 с весом $p=(+\infty, 1)$. Тогда по теореме 4 с этим весом выполняется равенство

$$
|Q-f|_{p, \Delta}=B_{1}:=\inf \left\{|P-f|_{p, \Delta}: P \in \mathscr{P}_{n}\right\} .
$$

Так как в случае веса $p=(+\infty, 1)$ для полиномов $P \in \mathscr{P}_{n}$ с $P(x) \geqslant f(x), x \in \Delta$, величина $|P-f|_{p, \Delta}$ конечна, то $B_{1}$, а поэтому и $|Q-f|_{p, \Delta}$ конечна; отсюда получим $Q(x) \geqslant f(x)$ при всех $x \in \Delta$. Для доказательства равенства (25) заметим, что

$$
B_{1}=B_{2}:=\inf \left\{|P-f|_{p, \Delta}: P \in \mathscr{P}_{n}, P(x) \geqslant f(x), x \in \Delta\right\} ;
$$

но $B_{2}=B$, так как при $P(x) \geqslant f(x), x \in \Delta$, имеем

$$
|(P-f, p)(x)|=(P(x)-f(x)) p_{+}(x)=|P(x)-f(x)| .
$$

Следовательно, для рассматриваемого полинома $Q(x)$ получим

$$
\|Q-f\|_{\Delta}=|Q-f|_{p, \Delta}=B_{1}=B_{2}=B
$$

т.е. вьполняется условие (25).

Обратно, пусть для некоторого полинома $Q \in \mathscr{P}_{n}$ выполнено условие (25) (вместе с $Q(x) \geqslant f(x)$ при $x \in \Delta)$. Тогда при $p=(+\infty, 1)$ имеем

$$
|Q-f|_{p, \Delta}=\|Q-f\|_{\Delta}=B=B_{2}=B_{1} .
$$

Поэтому по теореме 4 выполняется хотя бы одно из условий 1$)-3)$. Покажем, что условия 1) и 2) не выполняются. В нашем случае, очевидно, имеем $\Delta_{-}^{\infty}=\Delta$, $\Delta_{+}^{\infty}=\varnothing, \Delta\left(p_{-}\right)=\varnothing, \Delta\left(p_{-}, n\right) \subset \Delta_{-}^{\infty}(Q=f), \Delta\left(p_{+}, n\right)=\varnothing, \Delta_{+}^{\infty}(Q=f)=\varnothing$, поэтому $G_{-}=\Delta_{-}^{\infty}(Q=f)=\{x \in \Delta: Q(x)=f(x)\}, G_{+}=\Delta\left(p_{+}\right)$. В силу непрерывности $Q(x)$ и $f(x)$ множество $G_{-}$замкнуто и может содержать лишь точки $x \in \Delta$ с $Q(x)=f(x)$, поэтому $E_{-}=G_{-}$. В то же время множество $E_{+}=G_{+}=\Delta\left(p_{+}\right)$состоит из точек максимума $Q(x)-f(x)$ на $\Delta$ и может содержать лишь точки $x \in \Delta$ с $Q(x)>f(x)$.

Следовательно, $E_{-} \cap E_{+}=\varnothing$ и условие 1$)$ теоремы 4 не выполняется.

В случае веса $p=(+\infty, 1)$ условие 2$)$ теоремы 4 также не может выполняться, так как $\Delta_{+}^{\infty}=\varnothing$, а точки $p$-экстремума полной степени должны по определению принадлежать множеству $F=\Delta_{-}^{\infty} \cap \Delta_{+}^{\infty}$.

Следовательно, в случае веса $p=(+\infty, 1)$ выполнение равенства $(25)$ равносильно условию 3 ) теоремы 4. Отметим также, что индекс альтернанса в этом случае имеет вид $N_{n}(Q, f, p, \Delta)=1+m_{0}$, так как множество $F=\varnothing$.

Основной результат работы анонсирован в [6].

\section{Список литературы}

1. Долженко Е.П., Севастьлнов Е.А. Аппроксимации со знакочувствительньпм весом (теоремы существования и единственности) // Изв. РАН. Сер. матем. 1998. Т. 62. №6. C. 59-102.

2. Долженко Е. П., Севастьянов Е.А. Аппроксимация со знакочувствительньм весом (устойчивость, приложения к теории ужей и хаусдорфовьм аппроксимациям) // Изв. РАН. Сер. матем. 1999. Т. 63. №3. С. 77-118. 
3. Minkowsky H. Theorie der konvexen Korper, insbesondere Begrundung ihres Oberflachenbegriffs // Ges. Abh. 1911. V. 2. P. 131-229.

4. Крейн М. Г., Нудельман А. А. Проблема моментов Маркова и экстремальњые задачи. М.: Наука, 1973.

5. Рамазанов А.-Р. К., Рамазанов З.А. Критерии элемента наилучшего приближения для знакочувствительных аппроксимаций // Современные методы теории функций и смежные проблемы. Тезисы докладов Воронежской зимней математической школы. Воронеж, 2001. C. 219-220.

6. Рамазанов A.-P. К. Характеризация полинома наилучшего приближения непрерывной функции в несимметричной метрике // Функциональные пространства. Дифференциальные операторы. Проблемы математического образования. Международная конференция, посвященная Л. Д. Кудрявцеву. М.: Физматлит, 2003. С. 91-93.

Дагестанский государственньй университет

Поступила в редакцию 29.06 .2003 и 12.04 .2004 